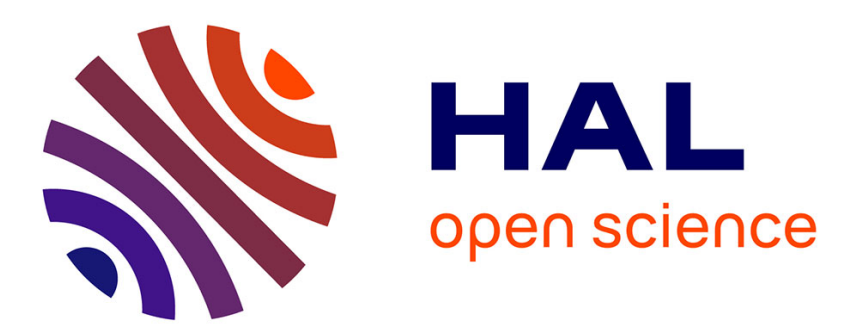

\title{
An unfitted Hybrid High-Order method for the Stokes interface problem
}

Erik Burman, Guillaume Delay, Alexandre Ern

\section{To cite this version:}

Erik Burman, Guillaume Delay, Alexandre Ern. An unfitted Hybrid High-Order method for the Stokes interface problem. IMA Journal of Numerical Analysis, 2021, 41 (4), pp.2362-2387. 10.1093/imanum/draa059 . hal-02519896v4

\section{HAL Id: hal-02519896 \\ https://hal.science/hal-02519896v4}

Submitted on 22 Jan 2021

HAL is a multi-disciplinary open access archive for the deposit and dissemination of scientific research documents, whether they are published or not. The documents may come from teaching and research institutions in France or abroad, or from public or private research centers.
L'archive ouverte pluridisciplinaire HAL, est destinée au dépôt et à la diffusion de documents scientifiques de niveau recherche, publiés ou non, émanant des établissements d'enseignement et de recherche français ou étrangers, des laboratoires publics ou privés. 


\title{
An unfitted hybrid high-order method for the Stokes interface problem
}

\author{
Erik Burman* \\ Guillaume Delay ${ }^{\dagger}$ \\ Alexandre Ern ${ }^{\ddagger}$
}

January 22, 2021

\begin{abstract}
We design and analyze a hybrid high-order (HHO) method on unfitted meshes to approximate the Stokes interface problem. The interface can cut through the mesh cells in a very general fashion. A cellagglomeration procedure prevents the appearance of small cut cells. Our main results are inf-sup stability and a priori error estimates with optimal convergence rates in the energy norm. Numerical simulations corroborate these results. Stokes interface problem, hybrid high-order method, unfitted meshes.
\end{abstract}

\section{Introduction}

Generating meshes to solve problems posed on domains with a curved interface separating subdomains with different properties can be a difficult task. The use of unfitted meshes that do not fit the interface greatly simplifies the meshing process since such meshes can be chosen in a very simple manner. We can for instance mesh the domain without taking into account the interface. The analysis of finite element methods (FEM) on unfitted meshes was started in $[2,3]$. The main paradigm introduced in [23] is to double the unknowns in the cut cells and to use Nitsche's method (see [31]) to weakly impose the interface conditions. We refer the reader to [10] for an overview. One difficulty with the penalty method appears with the presence of small cuts, i.e. cells that have only a tiny fraction of their volume on one side of the interface. Small cuts have an adverse effect on the conditioning of the system matrix and can even hamper convergence (see [17] for a recent discussion on this topic). There are essentially two ways to cure the issue of small cuts: one can either add some stabilization terms such as the ghost penalty technique devised in [8] or one can agglomerate cells in the vicinity of small cuts as in $[28,34]$.

In the present work we deal with the Stokes interface problem. Let $\Omega$ be a polygonal/polyhedral domain in $\mathbb{R}^{d}, d \in\{2,3\}$ (open, bounded, connected, Lipschitz subset of $\mathbb{R}^{d}$ ) and consider a partition of $\Omega$ into two disjoint subdomains so that $\bar{\Omega}=\overline{\Omega_{1}} \cup \overline{\Omega_{2}}$ with the interface $\Gamma=\partial \Omega_{1} \cap \partial \Omega_{2}$. The interface $\Gamma$ is assumed to be a smooth $(d-1)$-dimensional manifold of class $C^{2}$ that is not self-intersecting and that does not touch the boundary. These assumptions can be relaxed at the price of additional technicalities that are not further explored herein. One rather straightforward extension is to consider a piecewise smooth manifold. The unit normal vector $\boldsymbol{n}_{\Gamma}$ to $\Gamma$ conventionally points from $\Omega_{1}$ to $\Omega_{2}$. For a smooth enough function $v$ defined on $\Omega_{1} \cup \Omega_{2}$, we define its jump across $\Gamma$ as $\llbracket v \rrbracket_{\Gamma}:=v_{\mid \Omega_{1}}-v_{\mid \Omega_{2}}$. We denote $H^{1}\left(\Omega_{1} \cup \Omega_{2} ; \mathbb{R}^{d}\right):=\left\{\boldsymbol{v} \in L^{2}\left(\Omega ; \mathbb{R}^{d}\right) \mid \boldsymbol{v}_{\mid \Omega_{i}} \in H^{1}\left(\Omega_{i} ; \mathbb{R}^{d}\right), \forall i \in\{1,2\}\right\}$ and $L_{*}^{2}(\Omega):=\left\{q \in L^{2}(\Omega) \mid \int_{\Omega} q=0\right\}$. We consider the following problem: Find the velocity and pressure $(\boldsymbol{u}, p) \in H^{1}\left(\Omega_{1} \cup \Omega_{2} ; \mathbb{R}^{d}\right) \times L_{*}^{2}(\Omega)$ such that

$$
\begin{aligned}
& -\nabla \cdot \sigma(\boldsymbol{u}, p)=\boldsymbol{f}, \quad \nabla \cdot \boldsymbol{u}=0 \quad \text { in } \Omega_{1} \cup \Omega_{2}, \\
& \llbracket \sigma(\boldsymbol{u}, p) \rrbracket_{\Gamma} \boldsymbol{n}_{\Gamma}=\boldsymbol{g}_{N}, \quad \llbracket \boldsymbol{u} \rrbracket_{\Gamma}=\mathbf{0} \quad \text { on } \Gamma \\
& \boldsymbol{u}=\mathbf{0} \quad \text { on } \partial \Omega \text {, }
\end{aligned}
$$

with data $\boldsymbol{f} \in L^{2}\left(\Omega ; \mathbb{R}^{d}\right)$ and $\boldsymbol{g}_{N} \in L^{2}\left(\Gamma ; \mathbb{R}^{d}\right)$, and where $\sigma(\boldsymbol{u}, p):=2 \nu \nabla^{\mathrm{s}} \boldsymbol{u}-p \mathbb{I}$ is the total stress tensor, $\nabla^{\mathrm{s}} \boldsymbol{u}=\frac{1}{2}\left(\nabla \boldsymbol{u}+\nabla \boldsymbol{u}^{T}\right)$ is the linearized strain tensor, and $\mathbb{I}$ is the identity tensor. For simplicity we consider the viscosity $\nu$ to be constant in each subdomain $\Omega_{i}$ and we set $\nu_{i}:=\nu_{\mid \Omega_{i}}$ for all $i \in\{1,2\}$. To ensure robustness with respect to the viscosity contrast, the two subdomains play different roles in the numerical scheme. To fix the ideas, we enumerate the two subdomains so that $0<\nu_{1} \leq \nu_{2}$. We notice that several discretizations of the model problem (1) on unfitted meshes have already been analyzed, see for instance $[22,33,4,24,14,36,29,27,12,1]$

\footnotetext{
*Department of Mathematics, University College London, London, UK-WC1E 6BT, UK. Email: e.burman@ucl.ac.uk

†Sorbonne Université, CNRS, Université de Paris, LJLL, F-75005 Paris, France and Université Paris-Est, CERMICS (ENPC) and INRIA, Paris, France. Email: guillaume.delay@sorbonne-universite.fr

‡Université Paris-Est, CERMICS (ENPC), 77455 Marne-la-Vallée cedex 2, and INRIA, Paris, France. Email: alexandre.ern@enpc.fr
} 
for finite element discretizations and [34, 25] for discontinuous Galerkin. However among these works only [12] analyzes the approximation of (1) with high order polynomials.

The goal of the present work is to devise and analyze a hybrid high-order (HHO) method on unfitted meshes for the Stokes interface problem. Robustness with respect to small cuts is achieved by using a cell-agglomeration procedure. Let us notice that a polytopal method, as for instance the HHO method, is needed to deal with the general shape that can have the agglomerated cells. We also observe that in the case of a polygonal (i.e., piecewise flat) interface and provided the velocity does not jump across the interface as in (1b) (so that a single-valued unknown attached to the interface is meaningful), an alternative is to use a polytopal method on a fitted mesh. Thus one advantage of the present unfitted method is to deliver an effective approach to deal with (piecewise) curved interfaces. HHO methods have been introduced and analyzed for diffusion and locking-free linear elasticity problems on fitted meshes in [19,20]. As shown in [16], these methods are closely related to hybridizable discontinuous Galerkin (HDG) methods and to nonconforming virtual element methods (ncVEM). HHO methods have already been used to solve Stokes and Navier-Stokes problems on fitted meshes in [21, 6]. We consider an unfitted setting for HHO that has been first introduced and analyzed in [11] for an elliptic interface problem. This study has been continued in [9] where novel gradient reconstructions enabled the use of a parameter-free Nitsche's method to impose the interface conditions. Moreover, the unfitted HHO method has been run on several elliptic interface problems, including high contrast of coefficients and jumps of the solution across the interface. In the present work we extend the unfitted HHO method from [9] to the Stokes interface problem. The novelties herein are the devising of linearized strain and divergence reconstruction operators and the inf-sup stability analysis where we track the dependency on the viscosity coefficients on both sides of the interface and show that the method is robust in the highly contrasted case.

This work is organized as follows. We introduce the discretization of the model problem (1) by an unfitted HHO method in Section 2. We present the key analysis tools related to the discrete unfitted setting in Section 3. We perform the numerical analysis of the unfitted HHO method in Section 4, where we establish in particular inf-sup stability and a priori error estimates with optimal convergence rates. We then present some numerical examples in Section 5. Finally, conclusions are drawn in Section 6.

\section{The unfitted HHO method}

The goal of this section is to introduce some important definitions concerning unfitted meshes and to present the unfitted HHO method to discretize the Stokes interface problem.

\section{$2.1 \quad$ Unfitted meshes}

Let $\left(\mathcal{T}_{h}\right)_{h>0}$ be a family of matching meshes of $\Omega$. The meshes can have cells that are polyhedra in $\mathbb{R}^{d}$ with planar faces, and hanging nodes are also possible. For all $T \in \mathcal{T}_{h}, h_{T}$ denotes the diameter of the cell $T$ and $\boldsymbol{n}_{T}$ is the unit normal on $\partial T$ pointing outward $T$. We set $h:=\max _{T \in \mathcal{T}_{h}} h_{T}$. The mesh faces are collected in the set $\mathcal{F}_{h}$. Assumptions on the mesh regularity and how the interface cuts the mesh cells are stated in Section 3.1.

Let us define the partition $\mathcal{T}_{h}=\mathcal{T}_{h}^{1} \cup \mathcal{T}_{h}^{\Gamma} \cup \mathcal{T}_{h}^{2}$, where the subsets

$$
\mathcal{T}_{h}^{i}:=\left\{T \in \mathcal{T}_{h} \mid T \subset \Omega_{i}\right\} \quad(\forall i \in\{1,2\}), \quad \mathcal{T}_{h}^{\Gamma}:=\left\{T \in \mathcal{T}_{h} \mid T \cap \Gamma \neq \emptyset\right\},
$$

collect respectively the mesh cells inside the subdomain $\Omega_{i}$ (the uncut cells), and those cut by the interface $\Gamma$ (the cut cells). For every cut cell $T \in \mathcal{T}_{h}^{\Gamma}$ and all $i \in\{1,2\}$, we define

$$
T^{i}:=T \cap \Omega_{i}, \quad T^{\Gamma}:=T \cap \Gamma .
$$

For all $T \in \mathcal{T}_{h}^{\Gamma}$ and all $i \in\{1,2\}$, the boundary $\partial\left(T^{i}\right)$ of the subcell $T^{i}$ is decomposed as

$$
\partial\left(T^{i}\right)=(\partial T)^{i} \cup T^{\Gamma}, \quad(\partial T)^{i}:=\partial T \cap\left(\overline{\Omega_{i}} \backslash \Gamma\right) .
$$

In order to unify the notation, for all $i \in\{1,2\}$ and $T \in \mathcal{T}_{h}^{i}$, we also set

$$
T^{i}:=T, \quad T^{\bar{\imath}}:=\emptyset, \quad(\partial T)^{i}:=\partial T, \quad(\partial T)^{\bar{\imath}}:=\emptyset, \quad T^{\Gamma}:=\emptyset,
$$

where $\bar{\imath}:=3-i$ (so that $\overline{1}:=2$ and $\overline{2}:=1$ ). In a similar way, for all $F \in \mathcal{F}_{h}$ and all $i \in\{1,2\}$, we set

$$
F^{i}:=F \cap \Omega_{i} .
$$




\subsection{The local discrete problem}

In this section, we describe the unfitted HHO method for the Stokes interface problem. Let $k \geq 0$ be a polynomial degree. The discrete unknowns for the velocity are piecewise polynomials of degree $k$ attached to the mesh faces and of degree $(k+1)$ attached to the mesh cells, whereas the discrete unknowns for the pressure are piecewise polynomials of degree $k$ attached to the mesh cells. For any subset $S \subset \mathbb{R}^{d}$ consisting of one mesh (sub)cell or one mesh (sub)face, and for all $\ell \in \mathbb{N}$, we denote $\mathbb{P}^{\ell}(S)$ (resp. $\left.\mathbb{P}^{\ell}\left(S ; \mathbb{R}^{d}\right), \mathbb{P}^{\ell}\left(S ; \mathcal{S}^{d \times d}\right)\right)$ the space of scalar-valued (resp. vector-valued, symmetric matrix-valued) polynomials in $S$ of degree at most $\ell$. We also denote $(\cdot, \cdot)_{S}$ the $L^{2}$-scalar product on $S$ and $\|\cdot\|_{S}$ the associated norm. Whenever $S=\emptyset$, we abuse the notation by writing $\mathbb{P}^{\ell}(S):=\{0\}$ and $(\cdot, \cdot)_{S}:=0$.

Let $T \in \mathcal{T}_{h}$. For all $i \in\{1,2\}$, we set $\mathbb{P}^{k}\left(\mathcal{F}_{(\partial T)^{i}} ; \mathbb{R}^{d}\right):=X_{F \in \mathcal{F}_{(\partial T)^{i}}} \mathbb{P}^{k}\left(F ; \mathbb{R}^{d}\right)$ and $\mathcal{F}_{(\partial T)^{i}}:=\left\{F^{i} \mid F \in \mathcal{F}_{\partial T}\right\}$ where $\mathcal{F}_{\partial T}:=\left\{F \in \mathcal{F}_{h} \mid F \subset \partial T\right\}$. We define the local discrete unknowns as

$$
\begin{aligned}
\hat{\boldsymbol{v}}_{T} & :=\left(\boldsymbol{v}_{T^{1}}, \boldsymbol{v}_{(\partial T)^{1}}, \boldsymbol{v}_{T^{2}}, \boldsymbol{v}_{(\partial T)^{2}}\right) \in \hat{\boldsymbol{U}}_{T}^{k}, \\
p_{T} & :=\left(p_{T^{1}}, p_{T^{2}}\right) \in P_{T}^{k},
\end{aligned}
$$

with $\hat{\boldsymbol{U}}_{T}^{k}:=\hat{\boldsymbol{U}}_{T}^{k, 1} \times \hat{\boldsymbol{U}}_{T}^{k, 2}, P_{T}^{k}:=P_{T}^{k, 1} \times P_{T}^{k, 2}$, and

$$
\hat{\boldsymbol{U}}_{T}^{k, i}:=\mathbb{P}^{k+1}\left(T^{i} ; \mathbb{R}^{d}\right) \times \mathbb{P}^{k}\left(\mathcal{F}_{(\partial T)^{i}} ; \mathbb{R}^{d}\right), \quad P_{T}^{k, i}:=\mathbb{P}^{k}\left(T^{i}\right), \quad \forall i \in\{1,2\} .
$$

Note that there are no discrete unknowns attached to $T^{\Gamma}$. For all $T \in \mathcal{T}_{h}^{\Gamma}$ and all $\hat{\boldsymbol{v}}_{T} \in \hat{\boldsymbol{U}}_{T}$, we denote $\llbracket \boldsymbol{v}_{T} \rrbracket_{\Gamma}:=\boldsymbol{v}_{T^{1}}-\boldsymbol{v}_{T^{2}}$.

Remark 1 (Polynomial order). In the original HHO method from [19], equal-order unknowns are considered (i.e. polynomials of degree at most $k$ on faces and cells). In unfitted HHO methods it is also possible to consider equal-order unknowns in the uncut cells, but mixed-order unknowns are needed in the cut cells (i.e. polynomials of degree at most $k$ for the pressure cell unknowns and the velocity face unknowns and at most $(k+1)$ for the velocity cell unknowns). As discussed in [11] this is the prize to pay for avoiding unknowns on the curved interface and utilizing instead the trace of the cell unknowns. Note however that since the velocity cell unknowns are eliminated through static condensation, this choice does not increase the size of the discrete problem (see Section 2.3). For simplicity we consider in this work mixed-order unknowns in all the mesh cells.

For all $T \in \mathcal{T}_{h}$ and all $i \in\{1,2\}$, we define a symmetric gradient reconstruction operator $\mathbb{E}_{T^{i}}^{k}: \hat{\boldsymbol{U}}_{T}^{k} \rightarrow$ $\mathbb{P}^{k}\left(T^{i} ; \mathcal{S}^{d \times d}\right)$ such that for all $\hat{\boldsymbol{v}}_{T}:=\left(\boldsymbol{v}_{T^{1}}, \boldsymbol{v}_{(\partial T)^{1}}, \boldsymbol{v}_{T^{2}}, \boldsymbol{v}_{(\partial T)^{2}}\right) \in \hat{\boldsymbol{U}}_{T}^{k}$, we have

$$
\begin{aligned}
& \left(\mathbb{E}_{T^{1}}^{k}\left(\hat{\boldsymbol{v}}_{T}\right), \mathbb{q}\right)_{T^{1}}:=\left(\nabla^{\mathrm{s}} \boldsymbol{v}_{T^{1}}, \mathfrak{q}\right)_{T^{1}}+\left(\boldsymbol{v}_{(\partial T)^{1}}-\boldsymbol{v}_{T^{1}}, \mathbb{q} \boldsymbol{n}_{T}\right)_{(\partial T)^{1}}-\left(\llbracket \boldsymbol{v}_{T} \rrbracket_{\Gamma}, \mathbb{q} \boldsymbol{n}_{\Gamma}\right)_{T^{\Gamma}}, \\
& \left(\mathbb{E}_{T^{2}}^{k}\left(\hat{\boldsymbol{v}}_{T}\right), \mathbb{q}\right)_{T^{2}}:=\left(\nabla^{\mathrm{s}} \boldsymbol{v}_{T^{2}}, \mathfrak{q}\right)_{T^{2}}+\left(\boldsymbol{v}_{(\partial T)^{2}}-\boldsymbol{v}_{T^{2}}, \mathbb{q} \boldsymbol{n}_{T}\right)_{(\partial T)^{2}},
\end{aligned}
$$

for all $\mathbb{q} \in \mathbb{P}^{k}\left(T^{1} ; \mathcal{S}^{d \times d}\right)$ in (8a) and all $\mathbb{q} \in \mathbb{P}^{k}\left(T^{2} ; \mathcal{S}^{d \times d}\right)$ in (8b). Similarly, for all $T \in \mathcal{T}_{h}$ and all $i \in\{1,2\}$, we define a divergence reconstruction operator $D_{T^{i}}^{k}: \hat{\boldsymbol{U}}_{T}^{k} \rightarrow \mathbb{P}^{k}\left(T^{i}\right)$ such that for all $\hat{\boldsymbol{v}}_{T}:=$ $\left(\boldsymbol{v}_{T^{1}}, \boldsymbol{v}_{(\partial T)^{1}}, \boldsymbol{v}_{T^{2}}, \boldsymbol{v}_{(\partial T)^{2}}\right) \in \hat{\boldsymbol{U}}_{T}^{k}$, we have

$$
D_{T^{i}}^{k}\left(\hat{\boldsymbol{v}}_{T}\right):=\operatorname{trace}\left(\mathbb{E}_{T^{i}}^{k}\left(\hat{\boldsymbol{v}}_{T}\right)\right)
$$

i.e.,

$$
\begin{aligned}
\left(D_{T^{1}}^{k}\left(\hat{\boldsymbol{v}}_{T}\right), q\right)_{T^{1}}:=\left(\nabla \cdot \boldsymbol{v}_{T^{1}}, q\right)_{T^{1}}+\left(\boldsymbol{v}_{(\partial T)^{1}}-\boldsymbol{v}_{T^{1}}, q \boldsymbol{n}_{T}\right)_{(\partial T)^{1}}-\left(\llbracket \boldsymbol{v}_{T} \rrbracket_{\Gamma}, q \boldsymbol{n}_{\Gamma}\right)_{T^{\Gamma}}, \\
\left(D_{T^{2}}^{k}\left(\hat{\boldsymbol{v}}_{T}\right), q\right)_{T^{2}}:=\left(\nabla \cdot \boldsymbol{v}_{T^{2}}, q\right)_{T^{2}}+\left(\boldsymbol{v}_{(\partial T)^{2}}-\boldsymbol{v}_{T^{2}}, q \boldsymbol{n}_{T}\right)_{(\partial T)^{2}},
\end{aligned}
$$

for all $q \in \mathbb{P}^{k}\left(T^{1}\right)$ in (10a) and all $q \in \mathbb{P}^{k}\left(T^{2}\right)$ in (10b). Furthermore, the stabilization bilinear form is defined for all $\hat{\boldsymbol{v}}_{T}, \hat{\boldsymbol{w}}_{T} \in \hat{\boldsymbol{U}}_{T}^{k}$ as

$$
s_{T}\left(\hat{\boldsymbol{v}}_{T}, \hat{\boldsymbol{w}}_{T}\right):=\nu_{1} h_{T}^{-1}\left(\llbracket \boldsymbol{v}_{T} \rrbracket_{\Gamma}, \llbracket \boldsymbol{w}_{T} \rrbracket_{\Gamma}\right)_{T^{\Gamma}}+\sum_{i \in\{1,2\}} \nu_{i} h_{T}^{-1}\left(\Pi_{(\partial T)^{i}}^{k}\left(\boldsymbol{v}_{T^{i}}\right)-\boldsymbol{v}_{(\partial T)^{i}}, \Pi_{(\partial T)^{i}}^{k}\left(\boldsymbol{w}_{T^{i}}\right)-\boldsymbol{w}_{(\partial T)^{i}}\right)_{(\partial T)^{i}},
$$

where $\Pi_{(\partial T)^{i}}^{k}$ denotes the $L^{2}$-orthogonal projector onto $\mathbb{P}^{k}\left(\mathcal{F}_{(\partial T)^{i}} ; \mathbb{R}^{d}\right)$.

The local HHO bilinear and linear forms act as follows: For all $\left(\hat{\boldsymbol{v}}_{T}, r_{T}\right),\left(\hat{\boldsymbol{w}}_{T}, q_{T}\right) \in Y_{T}^{k}:=\hat{\boldsymbol{U}}_{T}^{k} \times P_{T}^{k}$,

$$
\begin{aligned}
A_{T}\left(\left(\hat{\boldsymbol{v}}_{T}, r_{T}\right),\left(\hat{\boldsymbol{w}}_{T}, q_{T}\right)\right):= & a_{T}\left(\hat{\boldsymbol{v}}_{T}, \hat{\boldsymbol{w}}_{T}\right)-b_{T}\left(\hat{\boldsymbol{w}}_{T}, r_{T}\right)+b_{T}\left(\hat{\boldsymbol{v}}_{T}, q_{T}\right) \\
& -\chi \nu_{2}^{-1} h_{T}\left(\llbracket \sigma\left(\boldsymbol{v}_{T}, r_{T}\right) \rrbracket_{\Gamma} \boldsymbol{n}_{\Gamma}, \llbracket \sigma\left(\boldsymbol{w}_{T},-q_{T}\right) \rrbracket_{\Gamma} \boldsymbol{n}_{\Gamma}\right)_{T^{\Gamma}}, \\
L_{T}\left(\hat{\boldsymbol{w}}_{T}, q_{T}\right):= & \ell_{T}\left(\hat{\boldsymbol{w}}_{T}\right)-\chi \nu_{2}^{-1} h_{T}\left(\boldsymbol{g}_{N}, \llbracket \sigma\left(\boldsymbol{w}_{T},-q_{T}\right) \rrbracket_{\Gamma} \boldsymbol{n}_{\Gamma}\right)_{T^{\Gamma}},
\end{aligned}
$$


with $\llbracket \sigma\left(\boldsymbol{v}_{T}, r_{T}\right) \rrbracket_{\Gamma}:=\sigma\left(\boldsymbol{v}_{T^{1}}, r_{T^{1}}\right)-\sigma\left(\boldsymbol{v}_{T^{2}}, r_{T^{2}}\right)$ and

$$
\begin{aligned}
a_{T}\left(\hat{\boldsymbol{v}}_{T}, \hat{\boldsymbol{w}}_{T}\right) & :=\sum_{i \in\{1,2\}} 2 \nu_{i}\left(\mathbb{E}_{T^{i}}^{k}\left(\hat{\boldsymbol{v}}_{T}\right), \mathbb{E}_{T^{i}}^{k}\left(\hat{\boldsymbol{w}}_{T}\right)\right)_{T^{i}}+s_{T}\left(\hat{\boldsymbol{v}}_{T}, \hat{\boldsymbol{w}}_{T}\right), \\
b_{T}\left(\hat{\boldsymbol{w}}_{T}, r_{T}\right) & :=\sum_{i \in\{1,2\}}\left(r_{T^{i}}, D_{T^{i}}^{k}\left(\hat{\boldsymbol{w}}_{T}\right)\right)_{T^{i}}, \\
\ell_{T}\left(\hat{\boldsymbol{w}}_{T}\right) & :=\sum_{i \in\{1,2\}}\left(\boldsymbol{f}, \boldsymbol{w}_{T^{i}}\right)_{T^{i}}+\left(\boldsymbol{g}_{N}, \boldsymbol{w}_{T^{2}}\right)_{T^{\Gamma}},
\end{aligned}
$$

and $\chi>0$ is a penalty parameter that has to be chosen small enough (see Lemma 10).

Remark 2 (Alternative gradient reconstruction). One can also consider a gradient reconstruction operator mapping to $\nabla^{\mathrm{s}} \mathbb{P}^{k}\left(T^{i} ; \mathbb{R}^{d}\right)$ defined in a similar way to the original HHO method in [19], for all $i \in\{1,2\}$. In this case, the specific divergence reconstruction defined in (10) has to be computed separately so as to evaluate the local bilinear form $b_{T}$ in (13b).

Remark 3 (Stabilization of pressure jumps). The role of the last term in (12a) is to provide a mild control on the pressure jumps across the interface, and the last term in (12b) is the consistent modification of the right-hand side. This control on the pressure jumps is needed in the present analysis to establish stability by means of an inf-sup condition (see Lemma 10). Our numerical tests indicate however that the stabilization term can be omitted (i.e., one can set $\chi:=0$ ). An inf-sup stability analysis with $\chi:=0$ has been carried out in [4] for a low-order unfitted finite element method, but extending this result to the present setting is an open question. Finally we observe that the symmetric gradient of the cell velocity is directly used to evaluate the jump of the stress tensor, without resorting to the symmetric gradient reconstruction. This is possible since we are using cell velocity unknowns of order $(k+1)$.

\subsection{The global discrete problem}

For all $i \in\{1,2\}$, we define the discrete spaces

$$
\hat{\boldsymbol{U}}_{h}^{k, i}:=\left(\mathrm{X}_{T \in \mathcal{T}_{h}} \mathbb{P}^{k+1}\left(T^{i} ; \mathbb{R}^{d}\right)\right) \times\left(\mathrm{X}_{F \in \mathcal{F}_{h}} \mathbb{P}^{k}\left(F^{i} ; \mathbb{R}^{d}\right)\right), \quad P_{h}^{k, i}:=\mathrm{X}_{T \in \mathcal{T}_{h}} \mathbb{P}^{k}\left(T^{i}\right),
$$

and we set

$$
\hat{\boldsymbol{U}}_{h}^{k}:=\hat{\boldsymbol{U}}_{h}^{k, 1} \times \hat{\boldsymbol{U}}_{h}^{k, 2}, \quad P_{h}^{k}:=P_{h}^{k, 1} \times P_{h}^{k, 2},
$$

as well as $P_{h *}^{k}:=\left\{q_{h} \in P_{h}^{k} \mid\left(q_{h}, 1\right)_{\Omega}=0\right\}$. We also denote $\hat{\boldsymbol{U}}_{h 0}^{k}$ the subspace of $\hat{\boldsymbol{U}}_{h}^{k}$ where all the degrees of freedom attached to the faces composing $\partial \Omega$ are null. Let $\hat{\boldsymbol{v}}_{h} \in \hat{\boldsymbol{U}}_{h}^{k}$ and let $q_{h} \in P_{h}^{k}$. For every cell $T \in \mathcal{T}_{h}$, we denote $\hat{\boldsymbol{v}}_{T}:=\left(\boldsymbol{v}_{T^{1}}, \boldsymbol{v}_{(\partial T)^{1}}, \boldsymbol{v}_{T^{2}}, \boldsymbol{v}_{(\partial T)^{2}}\right) \in \hat{\boldsymbol{U}}_{T}^{k}$ the components of $\hat{\boldsymbol{v}}_{h}$ attached to $T^{1}, T^{2}$ and the faces composing $(\partial T)^{1}$ and $(\partial T)^{2}$, and we denote $q_{T}:=\left(q_{T^{1}}, q_{T^{2}}\right) \in P_{T}^{k}$ the components of $q_{h}$ attached to $T^{1}$ and $T^{2}$ (see $\left.(7)\right)$.

The discrete problem reads as follows: Letting $Y_{h}^{k}:=\hat{\boldsymbol{U}}_{h 0}^{k} \times P_{h *}^{k}$, find $\left(\hat{\boldsymbol{u}}_{h}, p_{h}\right) \in Y_{h}^{k}$ such that

$$
A_{h}\left(\left(\hat{\boldsymbol{u}}_{h}, p_{h}\right),\left(\hat{\boldsymbol{w}}_{h}, q_{h}\right)\right)=L_{h}\left(\hat{\boldsymbol{w}}_{h}, q_{h}\right), \quad \forall\left(\hat{\boldsymbol{w}}_{h}, q_{h}\right) \in Y_{h}^{k},
$$

where for all $\left(\hat{\boldsymbol{v}}_{h}, r_{h}\right),\left(\hat{\boldsymbol{w}}_{h}, q_{h}\right) \in Y_{h}^{k}$,

$$
A_{h}\left(\left(\hat{\boldsymbol{v}}_{h}, r_{h}\right),\left(\hat{\boldsymbol{w}}_{h}, q_{h}\right)\right):=\sum_{T \in \mathcal{T}_{h}} A_{T}\left(\left(\hat{\boldsymbol{v}}_{T}, r_{T}\right),\left(\hat{\boldsymbol{w}}_{T}, q_{T}\right)\right), \quad L_{h}\left(\hat{\boldsymbol{w}}_{h}, q_{h}\right):=\sum_{T \in \mathcal{T}_{h}} L_{T}\left(\hat{\boldsymbol{w}}_{T}, q_{T}\right) .
$$

It is also convenient to define

$$
a_{h}\left(\hat{\boldsymbol{v}}_{h}, \hat{\boldsymbol{w}}_{h}\right):=\sum_{T \in \mathcal{T}_{h}} a_{T}\left(\hat{\boldsymbol{v}}_{T}, \hat{\boldsymbol{w}}_{T}\right), \quad b_{h}\left(\hat{\boldsymbol{v}}_{h}, q_{h}\right):=\sum_{T \in \mathcal{T}_{h}} b_{T}\left(\hat{\boldsymbol{v}}_{T}, q_{T}\right) .
$$

The discrete problem (16) can be solved efficiently by eliminating locally all the cell velocity unknowns and all the non constant pressure unknowns using static condensation. This local elimination leads to a global transmission problem involving only the velocity unknowns on the mesh skeleton and the mean pressure in every cell (one pressure degree of freedom per cell even in cut cells). The resulting stencil couples velocity unknowns attached to neighboring faces (in the sense of cells) and mean pressure values attached to neighboring cells (in the sense of faces). Once this global transmission problem is solved, the cell velocity and pressure unknowns are recovered by local solves (see [21]). 


\section{Analysis tools for unfitted meshes}

In this section we establish the key analysis tools on unfitted meshes regarding discrete inverse and multiplicative trace inequalities, as well as polynomial approximation properties.

\subsection{Admissible mesh sequences}

We consider a shape-regular polyhedral mesh sequence $\left(\mathcal{T}_{h}\right)_{h>0}$ in the sense of [19]. In a nutshell, any mesh $\mathcal{T}_{h}$ admits a matching simplicial submesh $\mathcal{T}_{h}^{\prime}$ such that any cell (or face) of $\mathcal{T}_{h}^{\prime}$ is a subset of a cell (or face) of $\mathcal{T}_{h}$. Moreover, there exists a mesh-regularity parameter $\rho>0$ such that for all $h>0$, all $T \in \mathcal{T}_{h}$, and all $S \in \mathcal{T}_{h}^{\prime}$ such that $S \subset T$, we have $\rho h_{S} \leq r_{S}$ and $\rho h_{T} \leq h_{S}$, where $r_{S}$ denotes the inradius of the simplex $S$. Notice that every mesh face is by assumption planar, but not necessarily connected. The geometric properties of the mesh sequence used in the analysis of unfitted methods are further discussed in [11, Section 6]. We also refer the reader to [18, Section 1.1] for a further discussion on shape-regular polyhedral mesh sequences.

In the context of unfitted methods, three additional assumptions on the meshes are needed. The first one quantifies how irregular the mesh cells can become due to the intersecting interface (and provides some discrete inverse inequalities), whereas the second one quantifies how well the mesh resolves the interface (and provides a multiplicative trace inequality) and the third one requires the meshes to be not too graded. Let $B(\boldsymbol{x}, r)$ be the ball of center $\boldsymbol{x} \in \mathbb{R}^{d}$ and radius $r>0$.

Assumption 1 (Cut cells). There is $\delta \in(0,1)$ such that, for all $T \in \mathcal{T}_{h}^{\Gamma}$ and all $i \in\{1,2\}$, there $i s \tilde{\boldsymbol{x}}_{T^{i}} \in T^{i}$ such that $B\left(\tilde{\boldsymbol{x}}_{T^{i}}, \delta h_{T}\right) \subset T^{i}$.

Assumption 2 (Resolving $\Gamma$ ). There is $\gamma \in(0,1)$ such that, for all $T \in \mathcal{T}_{h}^{\Gamma}$, there is a point $\check{\boldsymbol{x}}_{T} \in \mathbb{R}^{d}$ such that setting $T^{\dagger}:=B\left(\check{\boldsymbol{x}}_{T}, \gamma^{-1} h_{T}\right)$ we have the following properties: (i) $T \subset T^{\dagger}$; (ii) for all $\boldsymbol{s} \in T^{\Gamma}$, $d\left(\check{\boldsymbol{x}}_{T}, T_{\boldsymbol{s}} \Gamma\right) \geq \gamma h_{T}$, where $T_{\boldsymbol{s}} \Gamma$ is the tangent plane to $\Gamma$ at the point $\boldsymbol{s}$; (iii) For all $F \in \mathcal{F}_{\partial T}$, there is $\boldsymbol{x}_{F} \in T^{\dagger}$ such that $d\left(\boldsymbol{x}_{F}, F\right) \geq \gamma h_{T}$.

It is shown in $[11$, Lem. 6.4] that if the mesh is fine enough, it is possible to devise a two-step cellagglomeration procedure so that, choosing the parameter $\delta$ small enough (depending on the shape-regularity parameter $\rho$ ), Assumption 1 is fulfilled. In [9, Section 4.3], this procedure has been improved by adding a third step that guarantees that there is no propagation of the cell-agglomeration. Moreover it is shown in [11, Lem. 6.1] that if the mesh is fine enough compared to the curvature of the interface $\Gamma$, the statements (i) and (ii) in Assumption 2 are fulfilled, whereas the statement (iii) can be fulfilled by invoking the shape-regularity of the mesh sequence. Furthermore, on uncut cells, the shape regularity of the mesh sequence implies the existence of balls $B\left(\tilde{\boldsymbol{x}}_{T^{i}}, \delta h_{T}\right)$ (with $T^{i}=T$ ) and $T^{\dagger}:=B\left(\check{\boldsymbol{x}}_{T}, \gamma^{-1} h_{T}\right)$ satisfying the assertions of Assumptions 1 and 2 .

Finally, as in [9], we introduce a third assumption on the mesh sequence which is reasonable if the meshes are not excessively graded. For all $T \in \mathcal{T}_{h}$, let the neighboring layers $\Delta_{j}(T) \subset \mathbb{R}^{d}$ be defined by induction as $\Delta_{0}(T):=T$ and $\Delta_{j+1}(T):=\left\{T^{\prime} \in \mathcal{T}_{h} \mid \overline{T^{\prime}} \cap \overline{\Delta_{j}(T)} \neq \emptyset\right\}$ for all $j \in \mathbb{N}$.

Assumption 3 (Mild mesh grading). There is $n_{0} \in \mathbb{N}$ such that for all $T \in \mathcal{T}_{h}$, the ball $T^{\dagger}$ introduced in Assumption 2 satisfies $T^{\dagger} \subset \Delta_{n_{0}}(T)$.

\subsection{Discrete inverse and multiplicative trace inequalities}

The role of Assumption 1 is to provide the following discrete (inverse) inequalities.

Lemma 4 (Discrete (inverse) inequalities). Let Assumption 1 be fulfilled. Let $\ell \in \mathbb{N}$. There is $c_{\mathrm{disc}}>0$, depending on $\rho, \delta$, and $\ell$, such that, for all $T \in \mathcal{T}_{h}$, all $i \in\{1,2\}$ and all $v_{T^{i}} \in \mathbb{P}^{\ell}\left(T^{i}\right)$, the following inequalities hold true:

- (Discrete trace inequality) $\left\|v_{T^{i}}\right\|_{(\partial T)^{i} \cup T^{\Gamma}} \leq c_{\mathrm{disc}} h_{T}^{-\frac{1}{2}}\left\|v_{T^{i}}\right\|_{T^{i}}$.

- (Discrete inverse inequality) $\left\|\nabla v_{T^{i}}\right\|_{T^{i}} \leq c_{\mathrm{disc}} h_{T}^{-1}\left\|v_{T^{i}}\right\|_{T^{i}}$.

- (Discrete Poincaré inequality) $\left\|v_{T^{i}}\right\|_{T^{i}} \leq c_{\mathrm{disc}} h_{T}\left\|\nabla v_{T^{i}}\right\|_{T^{i}}$ whenever $\left(v_{T^{i}}, 1\right)_{B\left(\tilde{\boldsymbol{x}}_{T^{i}}, h_{T}\right)}=0$.

- (Discrete Korn's inequality) $\left\|\nabla v_{T^{i}}\right\|_{T^{i}} \leq c_{\mathrm{disc}}\left\|\nabla^{\mathrm{s}} v_{T^{i}}\right\|_{T^{i}}$ whenever $\left(v_{T^{i}}, r\right)_{B\left(\tilde{\boldsymbol{x}}_{T^{i}}, h_{T}\right)}=0$ for all $r \in \mathrm{RM}:=$ $\left\{r \in \mathbb{P}^{1}\left(\mathbb{R}^{d} ; \mathbb{R}^{d}\right) \mid \nabla^{\mathrm{s}} r=0\right\}$.

Proof. The discrete trace inequality is shown in [11, Lemma 3.4], and the discrete inverse and Poincaré inequalities are shown in [9, Lemma 3.4]. Let us now prove the discrete Korn's inequality. Let $T \in \mathcal{T}_{h}$ be a mesh cell. Invoking Korn's inequality in the ball $B\left(\tilde{\boldsymbol{x}}_{T^{i}}, h_{T}\right)$ (with constant $c_{0}$, see [26]) followed by the inverse inequality $\left\|\nabla^{\mathrm{s}} \boldsymbol{v}_{T^{i}}\right\|_{B\left(\tilde{\boldsymbol{x}}_{T^{i}}, h_{T}\right)} \leq c_{1}\left\|\nabla^{\mathrm{s}} \boldsymbol{v}_{T^{i}}\right\|_{B\left(\tilde{\boldsymbol{x}}_{T^{i}}, \delta h_{T}\right)}$ leads to $\left\|\nabla \boldsymbol{v}_{T^{i}}\right\|_{T^{i}} \leq\left\|\nabla \boldsymbol{v}_{T^{i}}\right\|_{B\left(\tilde{\boldsymbol{x}}_{T^{i}}, h_{T}\right)} \leq c_{0}\left\|\nabla^{\mathrm{s}} \boldsymbol{v}_{T^{i}}\right\|_{B\left(\tilde{\boldsymbol{x}}_{T^{i}}, h_{T}\right)} \leq$ $c_{0} c_{1}\left\|\nabla^{\mathrm{s}} \boldsymbol{v}_{T^{i}}\right\|_{B\left(\tilde{\boldsymbol{x}}_{T^{i}}, \delta h_{T}\right)} \leq c_{0} c_{1}\left\|\nabla^{\mathrm{s}} \boldsymbol{v}_{T^{i}}\right\|_{T^{i}}$ since $B\left(\tilde{\boldsymbol{x}}_{T^{i}}, \delta h_{T}\right) \subset T^{i} \subset B\left(\tilde{\boldsymbol{x}}_{T^{i}}, h_{T}\right)$. 
Remark 4 (Korn's inequality). The discrete Korn's inequality from Lemma 4 is established only for polynomial functions. This is sufficient for the present purpose where this inequality is needed only to prove the coercivity of the discrete bilinear form $a_{h}$. A Korn's inequality on polyhedral cells valid in the Sobolev space $H^{1}\left(T ; \mathbb{R}^{d}\right)$ is proven in [5, Appendix A.1].

The role of Assumption 2 is to provide the following multiplicative trace inequality (see [11] for the proof).

Lemma 5 (Multiplicative trace inequality). There is $c_{\mathrm{mtr}}>0$, depending on $\rho$ and $\gamma$, such that for all $T \in \mathcal{T}_{h}$, all $v \in H^{1}\left(T^{\dagger}\right)$, and all $i \in\{1,2\}$,

$$
\|v\|_{(\partial T)^{i} \cup T^{\Gamma}} \leq c_{\operatorname{mtr}}\left(h_{T}^{-\frac{1}{2}}\|v\|_{T^{\dagger}}+\|v\|_{T^{\dagger}}^{\frac{1}{2}}\|\nabla v\|_{T^{\dagger}}^{\frac{1}{2}}\right) .
$$

In what follows we use the convention $A \lesssim B$ to abbreviate the inequality $A \leq C B$ for positive real numbers $A$ and $B$, where the constant $C$ only depends on the polynomial degree $k \geq 0$ used in the unfitted HHO method, the mesh parameters $\rho, \delta, \gamma, n_{0}$ and the above constants $c_{\text {disc }}$ and $c_{\mathrm{mtr}}$, but does not depend neither on the viscosity coefficients $0<\nu_{1} \leq \nu_{2}$ nor on the mesh size $h>0$.

\subsection{Polynomial approximation}

Let $\boldsymbol{v} \in H^{1}\left(\Omega ; \mathbb{R}^{d}\right)$ and $q \in L^{2}(\Omega)$. To perform the error analysis, we assume that there is a real number $t \in\left(\frac{1}{2}, k+1\right]$ such that the exact solution satisfies $u_{\mid \Omega_{i}} \in H^{t+1}\left(\Omega_{i} ; \mathbb{R}^{d}\right)$ and $p_{\mid \Omega_{i}} \in H^{t}\left(\Omega_{i}\right)$ for all $i \in\{1,2\}$. Let $\boldsymbol{E}_{i}: H^{1+t}\left(\Omega_{i} ; \mathbb{R}^{d}\right) \rightarrow H^{1+t}\left(\mathbb{R}^{d} ; \mathbb{R}^{d}\right)$ and $E_{i}: H^{t}\left(\Omega_{i} ; \mathbb{R}\right) \rightarrow H^{t}\left(\mathbb{R}^{d} ; \mathbb{R}\right)$ be stable extension operators (see [13, 35]). For all $T \in \mathcal{T}_{h}$, recalling the spaces $\hat{\boldsymbol{U}}_{T}^{k}$ and $P_{T}^{k}$ from (7), we define for all $i \in\{1,2\}$,

$$
\begin{aligned}
& I_{T^{i}}^{k+1}(\boldsymbol{v}):=\Pi_{T^{\dagger}}^{k+1}\left(\boldsymbol{E}_{i}(\boldsymbol{v})\right)_{\mid T^{i}}, \quad \hat{I}_{T}^{k}(\boldsymbol{v}):=\left(I_{T^{1}}^{k+1}(\boldsymbol{v}), \Pi_{(\partial T)^{1}}^{k}(\boldsymbol{v}), I_{T^{2}}^{k+1}(\boldsymbol{v}), \Pi_{(\partial T)^{2}}^{k}(\boldsymbol{v})\right) \in \hat{\boldsymbol{U}}_{T}^{k}, \\
& J_{T^{i}}^{k}(q):=\Pi_{T^{\dagger}}^{k}\left(E_{i}(q)\right)_{\mid T^{i}},
\end{aligned}
$$

where $\Pi_{T^{\dagger}}^{k+1}$ and $\Pi_{T^{\dagger}}^{k}$ denote the $L^{2}$-orthogonal projectors onto $\mathbb{P}^{k+1}\left(T^{\dagger} ; \mathbb{R}^{d}\right)$ and $\mathbb{P}^{k}\left(T^{\dagger}\right)$, respectively, $T^{\dagger}$ is defined in Assumption 2, and $\Pi_{(\partial T)^{i}}^{k}$ is defined below (11). We also define $\hat{I}_{h}^{k}(\boldsymbol{v}) \in \hat{\boldsymbol{U}}_{h}^{k}$ such that, for all $T \in \mathcal{T}_{h}$, the local components of $\hat{I}_{h}^{k}(\boldsymbol{v})$ in $T$ are $\hat{I}_{T}^{k}(\boldsymbol{v}) \in \hat{\boldsymbol{U}}_{T}^{k}$. Note that $\hat{I}_{h}^{k}(\boldsymbol{v}) \in \hat{\boldsymbol{U}}_{h 0}^{k}$ whenever $\boldsymbol{v} \in H_{0}^{1}\left(\Omega ; \mathbb{R}^{d}\right)$.

Lemma 6 (Local approximation and global stability). Let $\boldsymbol{v} \in H^{1}\left(\Omega ; \mathbb{R}^{d}\right)$ and $q \in L^{2}(\Omega)$. For all $i \in\{1,2\}$, we assume that $\boldsymbol{v}_{\mid \Omega_{i}} \in H^{t+1}\left(\Omega_{i}\right)$ and $q_{\mid \Omega_{i}} \in H^{t}\left(\Omega_{i}\right)$. For all $T \in \mathcal{T}_{h}$ and all $i \in\{1,2\}$, we have

$$
\begin{aligned}
\left\|\boldsymbol{v}-I_{T^{i}}^{k+1}(\boldsymbol{v})\right\|_{T^{i}}+h_{T}^{\frac{1}{2}}\left\|\boldsymbol{v}-I_{T^{i}}^{k+1}(\boldsymbol{v})\right\|_{(\partial T)^{i} \cup T^{\Gamma}}+h_{T}\left|\boldsymbol{v}-I_{T^{i}}^{k+1}(\boldsymbol{v})\right|_{H^{1}\left(T^{i}\right)} & \lesssim h_{T}^{t+1}\left|\boldsymbol{E}_{i}(\boldsymbol{v})\right|_{H^{t+1}\left(T^{\dagger}\right)}, \\
\left\|J_{T^{i}}^{k}(q)-q\right\|_{T^{i}}+h_{T}^{\frac{1}{2}}\left\|J_{T^{i}}^{k}(q)-q\right\|_{(\partial T)^{i} \cup T^{\Gamma}} & \lesssim h_{T}^{t}\left|E_{i}(q)\right|_{H^{t}\left(T^{\dagger}\right)} .
\end{aligned}
$$

Moreover we have

$$
\begin{aligned}
& \sum_{T \in \mathcal{T}_{h}}\left|I_{T^{i}}^{k+1}(\boldsymbol{v})\right|_{H^{1}\left(T^{i}\right)}^{2} \lesssim|\boldsymbol{v}|_{H^{1}\left(\Omega_{i}\right)}^{2}, \\
& \sum_{T \in \mathcal{T}_{h}} \nu_{1} h_{T}^{-1}\left\|\llbracket \llbracket \boldsymbol{v}-I_{T}^{k+1}(\boldsymbol{v}) \rrbracket_{\Gamma}\right\|_{T^{\Gamma}}^{2}+\sum_{i \in\{1,2\}} \nu_{i} h_{T}^{-1}\left\|\Pi_{(\partial T)^{i}}^{k}(\boldsymbol{v})-I_{T^{i}}^{k+1}(\boldsymbol{v})\right\|_{(\partial T)^{i}}^{2} \lesssim \sum_{i \in\{1,2\}} \nu_{i}|\boldsymbol{v}|_{H^{1}\left(\Omega_{i}\right)}^{2} .
\end{aligned}
$$

Proof. The local approximation properties (21a)-(21b) follow from the approximation properties in $L^{2}$ and $H^{1}$ of the projectors $\Pi_{T^{\dagger}}^{k+1}$ and $\Pi_{T^{\dagger}}^{k}$ together with the multiplicative trace inequality from Lemma 5 . The global bound (22a) is a consequence of the $H^{1}$-stability of $\Pi_{T^{\dagger}}^{k+1}$, Assumption 3, and the $H^{1}$-stability of the extension operator $\boldsymbol{E}_{i}$ since we have

$$
\sum_{T \in \mathcal{T}_{h}}\left|I_{T^{i}}^{k+1}(\boldsymbol{v})\right|_{H^{1}\left(T^{i}\right)}^{2} \leq \sum_{T \in \mathcal{T}_{h}}\left|I_{T^{i}}^{k+1}(\boldsymbol{v})\right|_{H^{1}\left(T^{\dagger}\right)}^{2} \lesssim \sum_{T \in \mathcal{T}_{h}}\left|\boldsymbol{E}_{i}(\boldsymbol{v})\right|_{H^{1}\left(T^{\dagger}\right)}^{2} \lesssim\left|\boldsymbol{E}_{i}(\boldsymbol{v})\right|_{H^{1}\left(\mathbb{R}^{d}\right)}^{2} \lesssim|\boldsymbol{v}|_{H^{1}\left(\Omega_{i}\right)}^{2} .
$$

Finally the global bound (22b) follows by using similar arguments as above and $\nu_{1} \leq \nu_{2}$.

Lemma 7 (Approximation property of symmetric gradient reconstructions). Let $\boldsymbol{v} \in H^{1}\left(\Omega ; \mathbb{R}^{d}\right)$ be such that for all $i \in\{1,2\}, \boldsymbol{v}_{\mid \Omega_{i}} \in H^{t+1}\left(\Omega_{i} ; \mathbb{R}^{d}\right)$. For all $T \in \mathcal{T}_{h}$, we have

$$
\begin{aligned}
& \left\|\mathbb{E}_{T^{1}}^{k}\left(\hat{I}_{T}^{k}(\boldsymbol{v})\right)-\nabla^{\mathrm{s}} \boldsymbol{v}\right\|_{T^{1}}+h_{T}^{\frac{1}{2}}\left\|\mathbb{E}_{T^{1}}^{k}\left(\hat{I}_{T}^{k}(\boldsymbol{v})\right)-\nabla^{\mathrm{s}} \boldsymbol{v}\right\|_{(\partial T)^{1} \cup T^{\Gamma}} \lesssim h_{T}^{t} \sum_{i \in\{1,2\}}\left|\boldsymbol{E}_{i}(\boldsymbol{v})\right|_{H^{t+1}\left(T^{\dagger}\right)}, \\
& \left\|\mathbb{E}_{T^{2}}^{k}\left(\hat{I}_{T}^{k}(\boldsymbol{v})\right)-\nabla^{\mathrm{s}} \boldsymbol{v}\right\|_{T^{2}}+h_{T}^{\frac{1}{2}}\left\|\mathbb{E}_{T^{2}}^{k}\left(\hat{I}_{T}^{k}(\boldsymbol{v})\right)-\nabla^{\mathrm{s}} \boldsymbol{v}\right\|_{(\partial T)^{2} \cup T^{\Gamma}} \lesssim h_{T}^{t}\left|\boldsymbol{E}_{2}(\boldsymbol{v})\right|_{H^{t+1}\left(T^{\dagger}\right)} .
\end{aligned}
$$


Proof. We have

$$
\begin{aligned}
\left\|\mathbb{E}_{T^{1}}^{k}\left(\hat{I}_{T}^{k}(\boldsymbol{v})\right)-\nabla^{\mathrm{s}} I_{T^{1}}^{k+1}(\boldsymbol{v})\right\|_{T^{1}}^{2}= & -\left(\boldsymbol{v}-I_{T^{1}}^{k+1}(\boldsymbol{v}),\left(\nabla^{\mathrm{s}} I_{T^{1}}^{k+1}(\boldsymbol{v})-\mathbb{E}_{T^{1}}^{k}\left(\hat{I}_{T}^{k}(\boldsymbol{v})\right)\right) \boldsymbol{n}_{T}\right)_{(\partial T)^{1}} \\
& +\left(\llbracket I_{T}^{k+1}(\boldsymbol{v}) \rrbracket_{\Gamma},\left(\nabla^{\mathrm{s}} I_{T^{1}}^{k+1}(\boldsymbol{v})-\mathbb{E}_{T^{1}}^{k}\left(\hat{I}_{T}^{k}(\boldsymbol{v})\right)\right) \boldsymbol{n}_{\Gamma}\right)_{T^{\Gamma}},
\end{aligned}
$$

where we used the definition of $\mathbb{E}_{T^{1}}^{k}$ and the fact that $\mathbb{E}_{T^{1}}^{k}\left(\hat{I}_{T}^{k}(\boldsymbol{v})\right)-\nabla^{\mathrm{s}} I_{T^{1}}^{k+1}(\boldsymbol{v})$ belongs to $\mathbb{P}^{k}\left(T^{1} ; \mathcal{S}^{d \times d}\right)$ to replace $\Pi_{(\partial T)^{1}}^{k}(\boldsymbol{v})$ by $\boldsymbol{v}$. Invoking the Cauchy-Schwarz, the inverse trace, and the triangle inequalities and since $\llbracket \boldsymbol{v} \rrbracket_{\Gamma}=\mathbf{0}$ because $\boldsymbol{v} \in H^{1}\left(\Omega ; \mathbb{R}^{d}\right)$, we infer that

$$
\left\|\mathbb{E}_{T^{1}}^{k}\left(\hat{I}_{T}^{k}(\boldsymbol{v})\right)-\nabla^{\mathrm{s}} I_{T^{1}}^{k+1}(\boldsymbol{v})\right\|_{T^{1}} \lesssim h_{T}^{-\frac{1}{2}}\left(\left\|\boldsymbol{v}-I_{T^{1}}^{k+1}(\boldsymbol{v})\right\|_{(\partial T)^{1}}+\left\|\boldsymbol{v}-I_{T^{1}}^{k+1}(\boldsymbol{v})\right\|_{T^{\Gamma}}+\left\|\boldsymbol{v}-I_{T^{2}}^{k+1}(\boldsymbol{v})\right\|_{T^{\Gamma}}\right) .
$$

The local approximation property (21a) implies that

$$
\left\|\mathbb{E}_{T^{1}}^{k}\left(\hat{I}_{T}^{k}(\boldsymbol{v})\right)-\nabla^{\mathrm{s}} I_{T^{1}}^{k+1}(\boldsymbol{v})\right\|_{T^{1}} \lesssim h_{T}^{t} \sum_{i \in\{1,2\}}\left|\boldsymbol{E}_{i}(\boldsymbol{v})\right|_{H^{t+1}\left(T^{\dagger}\right)} .
$$

The discrete trace inequality from Lemma 4 also gives

$$
h_{T}^{\frac{1}{2}}\left\|\mathbb{E}_{T^{1}}^{k}\left(\hat{I}_{T}^{k}(\boldsymbol{v})\right)-\nabla^{\mathrm{s}} I_{T^{1}}^{k+1}(\boldsymbol{v})\right\|_{(\partial T)^{1} \cup T^{\Gamma}} \lesssim h_{T}^{t} \sum_{i \in\{1,2\}}\left|\boldsymbol{E}_{i}(\boldsymbol{v})\right|_{H^{t+1}\left(T^{\dagger}\right)} .
$$

Finally the triangle inequality applied to $\mathbb{E}_{T^{1}}^{k}\left(\hat{I}_{T}^{k}(\boldsymbol{v})\right)-\nabla^{\mathrm{s}} \boldsymbol{v}=\mathbb{E}_{T^{1}}^{k}\left(\hat{I}_{T}^{k}(\boldsymbol{v})\right)-\nabla^{\mathrm{s}} I_{T^{1}}^{k+1}(\boldsymbol{v})+\nabla^{\mathrm{s}}\left(I_{T^{1}}^{k+1}(\boldsymbol{v})-\boldsymbol{v}\right)$ together with the local $H^{1}$-approximation property (21a) yields (23a). The proof of (23b) uses similar arguments, but is simpler since we do not need to consider the jump across $\Gamma$.

\section{$4 \quad$ Stability and error analysis}

In this section we analyze the convergence of the unfitted HHO method for the Stokes interface problem. The proof consists in establishing stability together with consistency and boundedness properties. Note that most of the results in this section hold under the condition $k \geq 1$. This condition is indeed needed to utilize the discrete Korn's inequality which invokes an orthogonality property with respect to rigid body motions.

\subsection{Stability and well-posedness}

First we establish the coercivity and boundedness of the bilinear form $a_{h}$ related to the viscous term. The proofs are only sketched since they follow from the arguments from [20] in the fitted case and [11] in the unfitted one. We consider the following local semi-norm: For all $T \in \mathcal{T}_{h}$ and all $\hat{\boldsymbol{v}}_{T}:=\left(\boldsymbol{v}_{T^{1}}, \boldsymbol{v}_{(\partial T)^{1}}, \boldsymbol{v}_{T^{2}}, \boldsymbol{v}_{(\partial T)^{2}}\right) \in \hat{\boldsymbol{U}}_{T}^{k}$,

$$
\left|\hat{\boldsymbol{v}}_{T}\right|_{\boldsymbol{U}_{T}}^{2}:=\sum_{i \in\{1,2\}} \nu_{i}\left(\left\|\nabla^{\mathrm{s}} \boldsymbol{v}_{T^{i}}\right\|_{T^{i}}^{2}+h_{T}^{-1}\left\|\boldsymbol{v}_{T^{i}}-\boldsymbol{v}_{(\partial T)^{i}}\right\|_{(\partial T)^{i}}^{2}\right)+\nu_{1} h_{T}^{-1}\left\|\llbracket \boldsymbol{v}_{T} \rrbracket_{\Gamma}\right\|_{T^{\Gamma}}^{2},
$$

and $\left\|\hat{\boldsymbol{v}}_{h}\right\|_{\boldsymbol{U}_{h}}^{2}:=\sum_{T \in \mathcal{T}_{h}}\left|\hat{\boldsymbol{v}}_{T}\right|_{\boldsymbol{U}_{T}}^{2}$. Note that $\|\cdot\|_{\boldsymbol{U}_{h}}$ defines a norm on $\hat{\boldsymbol{U}}_{h 0}^{k}$. Indeed, if $\left\|\hat{\boldsymbol{v}}_{h}\right\|_{\boldsymbol{U}_{h}}=0$, then $\left|\hat{\boldsymbol{v}}_{T}\right|_{\boldsymbol{U}_{T}}=0$ for all $T \in \mathcal{T}_{h}$, which implies that $\boldsymbol{v}_{T^{i}}$ and $\boldsymbol{v}_{(\partial T)^{i}}$ take the same constant value for all $i \in\{1,2\}$ and that $\llbracket \boldsymbol{v}_{T} \rrbracket_{\Gamma}=\mathbf{0}$; we can then propagate the constant value of $\boldsymbol{v}_{T^{i_{0}}}=\boldsymbol{v}_{(\partial T)^{i_{0}}}$ up to the boundary $\partial \Omega$ (where $i_{0}$ is the index of the subdomain touching the boundary of $\Omega$ ) where $\boldsymbol{v}_{(\partial T)^{i}{ }_{0}}$ is zero by definition of $\hat{\boldsymbol{U}}_{h 0}^{k}$; finally, we use the zero jump condition across $\Gamma$ to propagate the zero value to the other subdomain.

Lemma 8 (Coercivity of $a_{h}$ ). Let $k \geq 1$. There exists $c_{\text {coer }}>0$ such that for all $\hat{\boldsymbol{v}}_{h} \in \hat{\boldsymbol{U}}_{h 0}^{k}$, the following holds true:

$$
c_{\text {coer }}\left\|\hat{\boldsymbol{v}}_{h}\right\|_{\boldsymbol{U}_{h}}^{2} \leq a_{h}\left(\hat{\boldsymbol{v}}_{h}, \hat{\boldsymbol{v}}_{h}\right) .
$$

Proof. Using the usual arguments one proves that

$$
\sum_{T \in \mathcal{T}_{h}} \nu_{1} h_{T}^{-1}\left\|\llbracket \boldsymbol{v}_{T} \rrbracket_{\Gamma}\right\|_{T^{\Gamma}}^{2}+\sum_{i \in\{1,2\}} \nu_{i}\left(\left\|\nabla^{\mathrm{s}} \boldsymbol{v}_{T^{i}}\right\|_{T^{i}}^{2}+h_{T}^{-1}\left\|\Pi_{(\partial T)^{i}}^{k}\left(\boldsymbol{v}_{T^{i}}\right)-\boldsymbol{v}_{(\partial T)^{i}}\right\|_{(\partial T)^{i}}^{2}\right) \lesssim a_{h}\left(\hat{\boldsymbol{v}}_{h}, \hat{\boldsymbol{v}}_{h}\right) .
$$

For $T \in \mathcal{T}_{h}$ and $i \in\{1,2\}$, to prove that $h_{T}^{-1}\left\|\left(I-\Pi_{(\partial T)^{i}}^{k}\right)\left(\boldsymbol{v}_{T^{i}}\right)\right\|_{(\partial T)^{i}}^{2} \lesssim\left\|\nabla^{\mathrm{s}} \boldsymbol{v}_{T^{i}}\right\|_{T^{i}}^{2}$, one observes that since $k \geq 1$,

$$
\begin{aligned}
h_{T}^{-1}\left\|\left(I-\Pi_{(\partial T)^{i}}^{k}\right)\left(\boldsymbol{v}_{T^{i}}\right)\right\|_{(\partial T)^{i}}^{2} & \leq h_{T}^{-1}\left\|\left(I-\Pi_{(\partial T)^{i}}^{k}\right)\left(\boldsymbol{v}_{T^{i}}-\Pi_{\mathrm{RM}}\left(\boldsymbol{v}_{T^{i}}\right)\right)\right\|_{(\partial T)^{i}}^{2} \\
& \leq h_{T}^{-1}\left\|\boldsymbol{v}_{T^{i}}-\Pi_{\mathrm{RM}}\left(\boldsymbol{v}_{T^{i}}\right)\right\|_{(\partial T)^{i}}^{2} \lesssim h_{T}^{-2}\left\|\boldsymbol{v}_{T^{i}}-\Pi_{\mathrm{RM}}\left(\boldsymbol{v}_{T^{i}}\right)\right\|_{T^{i}}^{2} \\
& \lesssim\left\|\nabla\left(\boldsymbol{v}_{T^{i}}-\Pi_{\mathrm{RM}}\left(\boldsymbol{v}_{T^{i}}\right)\right)\right\|_{T^{i}}^{2} \lesssim\left\|\nabla^{\mathrm{s}}\left(\boldsymbol{v}_{T^{i}}-\Pi_{\mathrm{RM}}\left(\boldsymbol{v}_{T^{i}}\right)\right)\right\|_{T^{i}}^{2} \leq\left\|\nabla^{\mathrm{s}} \boldsymbol{v}_{T^{i}}\right\|_{T^{i}}^{2},
\end{aligned}
$$


where $\Pi_{\mathrm{RM}}\left(\boldsymbol{v}_{T^{i}}\right)$ is the $L^{2}$-orthogonal projection of $\boldsymbol{v}_{T^{i}}$ onto RM defined in Lemma 4 . The above argument used the discrete inverse, Poincaré and Korn's inequalities from Lemma 4.

Lemma 9 (Boundedness of $\left.a_{h}\right)$. Let $k \geq 0$. For all $\hat{\boldsymbol{v}}_{h}, \hat{\boldsymbol{w}}_{h} \in \hat{\boldsymbol{U}}_{h}^{k}$, the following holds true:

$$
a_{h}\left(\hat{\boldsymbol{v}}_{h}, \hat{\boldsymbol{w}}_{h}\right) \lesssim\left\|\hat{\boldsymbol{v}}_{h}\right\|_{\boldsymbol{U}_{h}}\left\|\hat{\boldsymbol{w}}_{h}\right\|_{\boldsymbol{U}_{h}}
$$

Proof. It suffices to prove that $a_{T}\left(\hat{\boldsymbol{v}}_{T}, \hat{\boldsymbol{w}}_{T}\right) \lesssim\left|\hat{\boldsymbol{v}}_{T}\right|_{\boldsymbol{U}_{T}}\left|\hat{\boldsymbol{w}}_{T}\right|_{\boldsymbol{U}_{T}}$ for all $T \in \mathcal{T}_{h}$. The Cauchy-Schwarz inequality and the $L^{2}$-stability of $\Pi_{(\partial T)^{i}}^{k}$ imply that $\left|s_{T}\left(\hat{\boldsymbol{v}}_{T}, \hat{\boldsymbol{w}}_{T}\right)\right| \leq\left|\hat{\boldsymbol{v}}_{T}\right|_{\boldsymbol{U}_{T}}\left|\hat{\boldsymbol{w}}_{T}\right|_{\boldsymbol{U}_{T}}$. Moreover the definition (8a) of the reconstructed symmetric gradient yields

$$
\begin{aligned}
\left\|\mathbb{E}_{T^{1}}^{k}\left(\hat{\boldsymbol{v}}_{T}\right)\right\|_{T^{1}}^{2}= & \left(\mathbb{E}_{T^{1}}^{k}\left(\hat{\boldsymbol{v}}_{T}\right), \mathbb{E}_{T^{1}}^{k}\left(\hat{\boldsymbol{v}}_{T}\right)\right)_{T^{1}} \\
= & \left(\nabla^{\mathrm{s}} \boldsymbol{v}_{T^{1}}, \mathbb{E}_{T^{1}}^{k}\left(\hat{\boldsymbol{v}}_{T}\right)\right)_{T^{1}}+\left(\boldsymbol{v}_{(\partial T)^{1}}-\boldsymbol{v}_{T^{1}}, \mathbb{E}_{T^{1}}^{k}\left(\hat{\boldsymbol{v}}_{T}\right) \boldsymbol{n}_{T}\right)_{(\partial T)^{1}}-\left(\llbracket \boldsymbol{v}_{T} \rrbracket_{\Gamma}, \mathbb{E}_{T^{1}}^{k}\left(\hat{\boldsymbol{v}}_{T}\right) \boldsymbol{n}_{\Gamma}\right)_{T^{\Gamma}} \\
\leq & \left\|\mathbb{E}_{T^{1}}^{k}\left(\hat{\boldsymbol{v}}_{T}\right)\right\|_{T^{1}}\left\|\nabla^{\mathrm{s}} \boldsymbol{v}_{T^{1}}\right\|_{T^{1}}+h_{T}^{\frac{1}{2}}\left\|\mathbb{E}_{T^{1}}^{k}\left(\hat{\boldsymbol{v}}_{T}\right)\right\|_{(\partial T)^{1}} h_{T}^{-\frac{1}{2}}\left\|\boldsymbol{v}_{(\partial T)^{1}}-\boldsymbol{v}_{T^{i}}\right\|_{(\partial T)^{1}} \\
& +h_{T}^{\frac{1}{2}}\left\|\mathbb{E}_{T^{1}}^{k}\left(\hat{\boldsymbol{v}}_{T}\right)\right\|_{T^{\Gamma}} h_{T}^{-\frac{1}{2}}\left\|\llbracket \boldsymbol{v}_{T} \rrbracket_{\Gamma}\right\|_{T^{\Gamma}} .
\end{aligned}
$$

Invoking the discrete trace inequality from Lemma 4 shows that $\nu_{1}\left\|\mathbb{E}_{T^{1}}^{k}\left(\hat{\boldsymbol{v}}_{T}\right)\right\|_{T^{1}}^{2} \lesssim\left|\hat{\boldsymbol{v}}_{T}\right|_{\boldsymbol{U}_{T}}^{2}$. A similar inequality is obtained for $\left\|\mathbb{E}_{T^{2}}^{k}\left(\hat{\boldsymbol{v}}_{T}\right)\right\|_{T^{2}}^{2}$.

We now define, for all $T \in \mathcal{T}_{h}$ and all $\left(\hat{\boldsymbol{v}}_{T}, p_{T}\right) \in Y_{T}^{k}$,

$$
\begin{aligned}
\left|\left(\hat{\boldsymbol{v}}_{T}, r_{T}\right)\right|_{Y_{T}}^{2}:= & \left|\hat{\boldsymbol{v}}_{T}\right|_{\boldsymbol{U}_{T}}^{2}+\sum_{i \in\{1,2\}} \nu_{i}^{-1}\left\|r_{T^{i}}\right\|_{T^{i}}^{2} \\
= & \sum_{i \in\{1,2\}}\left(\nu_{i}\left\|\nabla^{\mathrm{s}} \boldsymbol{v}_{T^{i}}\right\|_{T^{i}}^{2}+\nu_{i} h_{T}^{-1}\left\|\boldsymbol{v}_{T^{i}}-\boldsymbol{v}_{(\partial T)^{i}}\right\|_{(\partial T)^{i}}^{2}+\nu_{i}^{-1}\left\|r_{T^{i}}\right\|_{T^{i}}^{2}\right) \\
& +\nu_{1} h_{T}^{-1}\left\|\llbracket \boldsymbol{v}_{T} \rrbracket_{\Gamma}\right\|_{T^{\Gamma}}^{2} .
\end{aligned}
$$

Summing the local semi-norms over the cells, we define, for all $\left(\hat{\boldsymbol{v}}_{h}, r_{h}\right) \in Y_{h}^{k}$,

$$
\left\|\left(\hat{\boldsymbol{v}}_{h}, r_{h}\right)\right\|_{Y_{h}}^{2}:=\sum_{T \in \mathcal{T}_{h}}\left|\left(\hat{\boldsymbol{v}}_{T}, r_{T}\right)\right|_{Y_{T}}^{2}=\left\|\hat{\boldsymbol{v}}_{h}\right\|_{\boldsymbol{U}_{h}}^{2}+\sum_{T \in \mathcal{T}_{h}} \sum_{i \in\{1,2\}} \nu_{i}^{-1}\left\|r_{T^{i}}\right\|_{T^{i}}^{2} .
$$

Note that $\|\cdot\|_{Y_{h}}$ defines a norm on $Y_{h}^{k}$.

Lemma 10 (Inf-sup stability of $A_{h}$ ). Let $k \geq 1$. Assume that the penalty parameter $\chi>0$ is small enough so that $\chi \leq \frac{\min \left(1, c_{\mathrm{coer}}\right)}{16} c_{\mathrm{disc}}^{-2}$, where $c_{\mathrm{disc}}$ is the constant in the discrete trace inequality from Lemma 4 and $c_{\mathrm{coer}}$ is the coercivity constant from Lemma 8. There exists $\beta>0$, scaling linearly with the penalty parameter $\chi$, such that for all $\left(\hat{\boldsymbol{v}}_{h}, r_{h}\right) \in Y_{h}^{k}$, we have

$$
\beta\left\|\left(\hat{\boldsymbol{v}}_{h}, r_{h}\right)\right\|_{Y_{h}} \leq \sup _{\left(\hat{\boldsymbol{w}}_{h}, q_{h}\right) \in Y_{h}^{k} \backslash\{0\}} \frac{A_{h}\left(\left(\hat{\boldsymbol{v}}_{h}, r_{h}\right),\left(\hat{\boldsymbol{w}}_{h}, q_{h}\right)\right)}{\left\|\left(\hat{\boldsymbol{w}}_{h}, q_{h}\right)\right\|_{Y_{h}}} .
$$

Moreover the discrete problem (16) is well-posed.

Proof. Let $\left(\hat{\boldsymbol{v}}_{h}, r_{h}\right) \in Y_{h}^{k}$ and set $S:=\sup _{\left(\hat{\boldsymbol{w}}_{h}, q_{h}\right) \in Y_{h}^{k} \backslash\{0\}} \frac{A_{h}\left(\left(\hat{\boldsymbol{v}}_{h}, r_{h}\right),\left(\hat{\boldsymbol{w}}_{h}, q_{h}\right)\right)}{\left\|\left(\hat{\boldsymbol{w}}_{h}, q_{h}\right)\right\|_{Y_{h}}}$.

- Control on the velocity and pressure jumps. Recalling the definitions of $A_{h}$ and $A_{T}$ (see (17) and (12a)), we have

$$
A_{h}\left(\left(\hat{\boldsymbol{v}}_{h}, r_{h}\right),\left(\hat{\boldsymbol{v}}_{h}, r_{h}\right)\right)=a_{h}\left(\hat{\boldsymbol{v}}_{h}, \hat{\boldsymbol{v}}_{h}\right)+\chi \sum_{T \in \mathcal{T}_{h}} \nu_{2}^{-1} h_{T}\left(\left\|\llbracket r_{T} \rrbracket_{\Gamma}\right\|_{T^{\Gamma}}^{2}-\left\|\llbracket \sigma\left(\boldsymbol{v}_{T}, 0\right) \rrbracket_{\Gamma} \boldsymbol{n}_{\Gamma}\right\|_{T^{\Gamma}}^{2}\right) .
$$

We use the coercivity of $a_{h}$ (Lemma 8$)$ to infer that

$$
\begin{array}{r}
\min \left(1, c_{\text {coer }}\right)\left(\left\|\hat{\boldsymbol{v}}_{h}\right\|_{\boldsymbol{U}_{h}}^{2}+\chi \sum_{T \in \mathcal{T}_{h}} \nu_{2}^{-1} h_{T}\left\|\llbracket r_{T} \rrbracket_{\Gamma}\right\|_{T^{\Gamma}}^{2}\right) \leq a_{h}\left(\hat{\boldsymbol{v}}_{h}, \hat{\boldsymbol{v}}_{h}\right)+\chi \sum_{T \in \mathcal{T}_{h}} \nu_{2}^{-1} h_{T}\left\|\llbracket r_{T} \rrbracket_{\Gamma}\right\|_{T^{\Gamma}}^{2} \\
\leq A_{h}\left(\left(\hat{\boldsymbol{v}}_{h}, r_{h}\right),\left(\hat{\boldsymbol{v}}_{h}, r_{h}\right)\right)+\chi \sum_{T \in \mathcal{T}_{h}} \nu_{2}^{-1} h_{T}\left\|\llbracket \sigma\left(\boldsymbol{v}_{T}, 0\right) \rrbracket_{\Gamma} \boldsymbol{n}_{\Gamma}\right\|_{T^{\Gamma}}^{2} .
\end{array}
$$


Since $\nu_{1} \leq \nu_{2}$, we have $\sum_{T \in \mathcal{T}_{h}} \nu_{2}^{-1} h_{T}\left\|\llbracket \sigma\left(\boldsymbol{v}_{T}, 0\right) \rrbracket_{\Gamma} \boldsymbol{n}_{\Gamma}\right\|_{T^{\Gamma}}^{2} \leq 8 c_{\text {disc }}^{2}\left\|\hat{\boldsymbol{v}}_{h}\right\|_{\boldsymbol{U}_{h}}^{2}$ owing to the discrete trace inequality from Lemma 4 . Since $\chi \leq \frac{\min \left(1, c_{\text {coer }}\right)}{16} c_{\text {disc }}^{-2}$ by assumption, the rightmost term in the above right-hand side can be hidden on the left-hand side. We infer that

$$
\left\|\hat{\boldsymbol{v}}_{h}\right\|_{\boldsymbol{U}_{h}}^{2}+\chi \sum_{T \in \mathcal{T}_{h}} \nu_{2}^{-1} h_{T}\left\|\llbracket r_{T} \rrbracket_{\Gamma}\right\|_{T^{\Gamma}}^{2} \lesssim A_{h}\left(\left(\hat{\boldsymbol{v}}_{h}, r_{h}\right),\left(\hat{\boldsymbol{v}}_{h}, r_{h}\right)\right) \leq S\left\|\left(\hat{\boldsymbol{v}}_{h}, r_{h}\right)\right\|_{Y_{h}} .
$$

- Control on the pressure gradient. Let $\hat{\boldsymbol{w}}_{h} \in \hat{\boldsymbol{U}}_{h 0}^{k}$ be such that, for all $T \in \mathcal{T}_{h}, \hat{\boldsymbol{w}}_{T}:=\left(\boldsymbol{w}_{T^{1}}, \mathbf{0}, \boldsymbol{w}_{T^{2}}, \mathbf{0}\right)$ with $\boldsymbol{w}_{T^{i}}:=-\nu_{i}^{-1} h_{T}^{2} \nabla r_{T^{i}}$. We have

$$
\begin{aligned}
h_{T}^{2} \sum_{i \in\{1,2\}} \nu_{i}^{-1}\left\|\nabla r_{T^{i}}\right\|_{T^{i}}^{2} & =-\sum_{i \in\{1,2\}}\left(\nabla r_{T^{i}}, \boldsymbol{w}_{T^{i}}\right)_{T^{i}} \\
& =\left(r_{T^{2}} \boldsymbol{n}_{\Gamma}, \boldsymbol{w}_{T^{2}}\right)_{T^{\Gamma}}-\left(r_{T^{1}} \boldsymbol{n}_{\Gamma}, \boldsymbol{w}_{T^{1}}\right)_{T^{\Gamma}}+\sum_{i \in\{1,2\}}\left(\left(r_{T^{i}}, \nabla \cdot \boldsymbol{w}_{T^{i}}\right)_{T^{i}}-\left(r_{T^{i}} \boldsymbol{n}_{T}, \boldsymbol{w}_{T^{i}}\right)_{(\partial T)^{i}}\right) \\
& =\sum_{i \in\{1,2\}}\left(r_{T^{i}}, D_{T^{i}}^{k}\left(\hat{\boldsymbol{w}}_{T}\right)\right)_{T^{i}}-\left(\llbracket r_{T} \rrbracket_{\Gamma} \boldsymbol{n}_{\Gamma}, \boldsymbol{w}_{T^{2}}\right)_{T^{\Gamma}} .
\end{aligned}
$$

We infer that

$$
\begin{aligned}
\sum_{T \in \mathcal{T}_{h}} h_{T}^{2} \sum_{i \in\{1,2\}} \nu_{i}^{-1}\left\|\nabla r_{T^{i}}\right\|_{T^{i}}^{2}= & b_{h}\left(\hat{\boldsymbol{w}}_{h}, r_{h}\right)-\sum_{T \in \mathcal{T}_{h}}\left(\llbracket r_{T} \rrbracket_{\Gamma} \boldsymbol{n}_{\Gamma}, \boldsymbol{w}_{T^{2}}\right)_{T^{\Gamma}} \\
= & -A_{h}\left(\left(\hat{\boldsymbol{v}}_{h}, r_{h}\right),\left(\hat{\boldsymbol{w}}_{h}, 0\right)\right)+a_{h}\left(\hat{\boldsymbol{v}}_{h}, \hat{\boldsymbol{w}}_{h}\right)-\sum_{T \in \mathcal{T}_{h}}\left(\llbracket r_{T} \rrbracket_{\Gamma} \boldsymbol{n}_{\Gamma}, \boldsymbol{w}_{T^{2}}\right)_{T^{\Gamma}} \\
& -\chi \sum_{T \in \mathcal{T}_{h}} \nu_{2}^{-1} h_{T}\left(\llbracket \sigma\left(\boldsymbol{v}_{T}, r_{T}\right) \rrbracket_{\Gamma} \boldsymbol{n}_{\Gamma}, \llbracket \sigma\left(\boldsymbol{w}_{T}, 0\right) \rrbracket_{\Gamma} \boldsymbol{n}_{\Gamma}\right)_{T^{\Gamma}} .
\end{aligned}
$$

Let us denote $A_{1}, A_{2}, A_{3}$, and $A_{4}$ the four terms on the right-hand side. We have $\left|A_{1}\right| \leq S\left\|\hat{\boldsymbol{w}}_{h}\right\|_{\boldsymbol{U}_{h}}$ by definition of $S$, and we have $\left|A_{2}\right| \lesssim\left\|\hat{\boldsymbol{v}}_{h}\right\|_{\boldsymbol{U}_{h}}\left\|\hat{\boldsymbol{w}}_{h}\right\|_{\boldsymbol{U}_{h}}$ owing to the boundedness of $a_{h}$ (Lemma 9). Moreover, the CauchySchwarz and Young inequalities, the discrete trace inequality from Lemma 4 and the definition of $\boldsymbol{w}_{T^{2}}$ imply that

$$
\left|A_{3}\right| \leq \alpha \sum_{T \in \mathcal{T}_{h}} h_{T^{2}}^{2} \nu_{2}^{-1}\left\|\nabla r_{T^{2}}\right\|_{T^{2}}^{2}+c_{\alpha} \sum_{T \in \mathcal{T}_{h}} \nu_{2}^{-1} h_{T}\left\|\llbracket r_{T} \rrbracket_{\Gamma}\right\|_{T^{\Gamma}}^{2}
$$

where $\alpha$ can be chosen as small as needed and $c_{\alpha}>0$. Finally, recalling the definition of $\sigma\left(\boldsymbol{v}_{T}, r_{T}\right)$, using that $\nu_{1} \leq \nu_{2}$, and invoking again the discrete trace inequality from Lemma 4 leads to

$$
\left|A_{4}\right| \lesssim\left(\left\|\hat{\boldsymbol{v}}_{h}\right\|_{\boldsymbol{U}_{h}}^{2}+\sum_{T \in \mathcal{T}_{h}} \nu_{2}^{-1} h_{T}\left\|\llbracket r_{T} \rrbracket_{\Gamma}\right\|_{T^{\Gamma}}^{2}\right)^{\frac{1}{2}}\left\|\hat{\boldsymbol{w}}_{h}\right\|_{\boldsymbol{U}_{h}} .
$$

Putting everything together and choosing $\alpha$ small enough, we infer that

$$
\sum_{T \in \mathcal{T}_{h}} h_{T}^{2} \sum_{i \in\{1,2\}} \nu_{i}^{-1}\left\|\nabla r_{T^{i}}\right\|_{T^{i}}^{2} \lesssim S^{2}+\left\|\hat{\boldsymbol{v}}_{h}\right\|_{\boldsymbol{U}_{h}}^{2}+\sum_{T \in \mathcal{T}_{h}} \nu_{2}^{-1} h_{T}\left\|\llbracket r_{T} \rrbracket_{\Gamma}\right\|_{T^{\Gamma}}^{2}
$$

Combined with (30) and since $\chi \lesssim 1$, the above bound implies that

$$
\sum_{T \in \mathcal{T}_{h}} h_{T}^{2} \sum_{i \in\{1,2\}} \nu_{i}^{-1}\left\|\nabla r_{T^{i}}\right\|_{T^{i}}^{2} \lesssim S^{2}+\chi^{-1} S\left\|\left(\hat{\boldsymbol{v}}_{h}, r_{h}\right)\right\|_{Y_{h}} .
$$

- Control on the pressure in the $L^{2}$-norm. Letting $\kappa:=\frac{1}{|\Omega|} \sum_{T \in \mathcal{T}_{h}} \sum_{i \in\{1,2\}} \nu_{i}^{-1}\left(r_{T^{i}}, 1\right)_{T^{i}}$, there exists $\boldsymbol{v}_{r_{h}} \in$ $H_{0}^{1}(\Omega)$ such that $\nabla \cdot \boldsymbol{v}_{r_{h}}=\nu^{-1} r_{h}-\kappa$ in $\Omega$, i.e. $\left(\nabla \cdot \boldsymbol{v}_{r_{h}}\right)_{\mid T^{i}}=\nu_{i}^{-1} r_{T^{i}}-\kappa$ for all $T \in \mathcal{T}_{h}$ and all $i \in\{1,2\}$, and we also have $\sum_{i \in\{1,2\}}\left\|\boldsymbol{v}_{r_{h}}\right\|_{H^{1}\left(\Omega_{i}\right)} \lesssim \sum_{i \in\{1,2\}} \nu_{i}^{-1}\left(\sum_{T \in \mathcal{T}_{h}}\left\|r_{T^{i}}\right\|_{T^{i}}^{2}\right)^{\frac{1}{2}}$. Let $\hat{\boldsymbol{w}}_{h}:=\hat{I}_{h}^{k}\left(\boldsymbol{v}_{r_{h}}\right)$ and note that $\hat{\boldsymbol{w}}_{h} \in \hat{\boldsymbol{U}}_{h 0}^{k}$. We have

$$
\sum_{T \in \mathcal{T}_{h}} \sum_{i \in\{1,2\}} \nu_{i}^{-1}\left\|r_{T^{i}}\right\|_{T^{i}}^{2}=\sum_{T \in \mathcal{T}_{h}} \sum_{i \in\{1,2\}}\left(r_{T^{i}}, \nabla \cdot \boldsymbol{v}_{r_{h}}\right)_{T^{i}}
$$

since $\kappa \sum_{T \in \mathcal{T}_{h}} \sum_{i \in\{1,2\}}\left(r_{T^{i}}, 1\right)_{T^{i}}=0$ because $r_{h} \in P_{h *}^{k}$. Therefore we can write $\sum_{T \in \mathcal{T}_{h}} \sum_{i \in\{1,2\}} \nu_{i}^{-1}\left\|r_{T^{i}}\right\|_{T^{i}}^{2}=$ $\Psi_{1}+\Psi_{2}$ with

$$
\Psi_{1}:=\sum_{T \in \mathcal{T}_{h}} \sum_{i \in\{1,2\}}\left(r_{T^{i}}, \nabla \cdot \boldsymbol{v}_{r_{h}}-D_{T^{i}}^{k}\left(\hat{\boldsymbol{w}}_{T}\right)\right)_{T^{i}}, \quad \Psi_{2}:=\sum_{T \in \mathcal{T}_{h}} \sum_{i \in\{1,2\}}\left(r_{T^{i}}, D_{T^{i}}^{k}\left(\hat{\boldsymbol{w}}_{T}\right)\right)_{T^{i}}=b_{h}\left(\hat{\boldsymbol{w}}_{h}, r_{h}\right) .
$$


Incidentally we notice that $\Psi_{1}=0$ in the absence of cut cells (as in the standard fitted HHO method for the Stokes equations). We observe that

$$
\begin{aligned}
& \Psi_{1}= \sum_{T \in \mathcal{T}_{h}}\left(\llbracket \boldsymbol{w}_{T} \rrbracket_{\Gamma}, r_{T^{1}} \boldsymbol{n}_{\Gamma}\right)_{T^{\Gamma}}+\sum_{i \in\{1,2\}}\left(\left(r_{T^{i}}, \nabla \cdot\left(\boldsymbol{v}_{r_{h}}-\boldsymbol{w}_{T^{i}}\right)\right)_{T^{i}}+\left(r_{T^{i}} \boldsymbol{n}_{T}, \boldsymbol{w}_{T^{i}}-\boldsymbol{w}_{(\partial T)^{i}}\right)_{(\partial T)^{i}}\right) \\
&=\sum_{T \in \mathcal{T}_{h}}-\left(r_{T^{1}} \boldsymbol{n}_{\Gamma}, \boldsymbol{w}_{T^{1}}-\boldsymbol{v}_{r_{h}}\right)_{T^{\Gamma}}+\left(r_{T^{2}} \boldsymbol{n}_{\Gamma}, \boldsymbol{w}_{T^{2}}-\boldsymbol{v}_{r_{h}}\right)_{T^{\Gamma}}+\left(\llbracket \boldsymbol{w}_{T} \rrbracket_{\Gamma}, r_{T^{1}} \boldsymbol{n}_{\Gamma}\right)_{T^{\Gamma}} \\
& \quad+\sum_{i \in\{1,2\}}\left(-\left(\nabla r_{T^{i}}, \boldsymbol{v}_{r_{h}}-\boldsymbol{w}_{T^{i}}\right)_{T^{i}}+\left(r_{T^{i}} \boldsymbol{n}_{T}, \boldsymbol{v}_{r_{h}}-\boldsymbol{w}_{(\partial T)^{i}}\right)_{(\partial T)^{i}}\right) \\
& \quad \\
& \quad \sum_{T \in \mathcal{T}_{h}}-\left(\boldsymbol{w}_{T^{2}}-\boldsymbol{v}_{r_{h}}, \llbracket r_{T} \rrbracket_{\Gamma} \boldsymbol{n}_{\Gamma}\right)_{T^{\Gamma}}+\sum_{i \in\{1,2\}}\left(-\left(\nabla r_{T^{i}}, \boldsymbol{v}_{r_{h}}-\boldsymbol{w}_{T^{i}}\right)_{T^{i}}+\left(r_{T^{i}} \boldsymbol{n}_{T}, \boldsymbol{v}_{r_{h}}-\boldsymbol{w}_{(\partial T)^{i}}\right)_{(\partial T)^{i}}\right) .
\end{aligned}
$$

We have $\left(r_{T^{i}} \boldsymbol{n}_{T}, \boldsymbol{v}_{r_{h}}-\boldsymbol{w}_{(\partial T)^{i}}\right)_{(\partial T)^{i}}=0$ and the approximation properties of $\boldsymbol{w}_{T^{i}}$ (Lemma 6) give

$$
\begin{aligned}
\left|\Psi_{1}\right| & \lesssim \sum_{T \in \mathcal{T}_{h}}\left\|\boldsymbol{w}_{T^{2}}-\boldsymbol{v}_{r_{h}}\right\|_{T^{\Gamma}}\left\|\llbracket r_{T} \rrbracket_{\Gamma}\right\|_{T^{\Gamma}}+\sum_{i \in\{1,2\}}\left\|\nabla r_{T^{i}}\right\|_{T^{i}}\left\|\boldsymbol{v}_{r_{h}}-I_{T^{i}}^{k+1}\left(\boldsymbol{v}_{r_{h}}\right)\right\|_{T^{i}} \\
& \lesssim\left(\left(\sum_{T \in \mathcal{T}_{h}} \nu_{2}^{-1} h_{T}\left\|\llbracket r_{T} \rrbracket_{\Gamma}\right\|_{T^{\Gamma}}^{2}\right)^{\frac{1}{2}}+\left(\sum_{T \in \mathcal{T}_{h}} \sum_{i \in\{1,2\}} \nu_{i}^{-1} h_{T^{2}}^{2}\left\|\nabla r_{T^{i}}\right\|_{T^{i}}^{2}\right)^{\frac{1}{2}}\right)\left(\sum_{T \in \mathcal{T}_{h}} \sum_{i \in\{1,2\}} \nu_{i}\left|\boldsymbol{E}_{i}\left(\boldsymbol{v}_{r_{h}}\right)\right|_{H^{1}\left(T^{\dagger}\right)}^{2}\right)^{\frac{1}{2}}
\end{aligned}
$$

Moreover by definition of the bilinear form $A_{h}$, we have

$$
\Psi_{2}=-A_{h}\left(\left(\hat{\boldsymbol{v}}_{h}, r_{h}\right),\left(\hat{\boldsymbol{w}}_{h}, 0\right)\right)+a_{h}\left(\hat{\boldsymbol{v}}_{h}, \hat{\boldsymbol{w}}_{h}\right)-\chi \sum_{T \in \mathcal{T}_{h}} \nu_{2}^{-1} h_{T}\left(\llbracket \sigma\left(\boldsymbol{v}_{T}, r_{T}\right) \rrbracket_{\Gamma} \boldsymbol{n}_{\Gamma}, \llbracket \sigma\left(\boldsymbol{w}_{T}, 0\right) \rrbracket_{\Gamma} \boldsymbol{n}_{\Gamma}\right)_{T^{\Gamma}},
$$

so that

$$
\left|\Psi_{2}\right| \lesssim S\left\|\hat{\boldsymbol{w}}_{h}\right\|_{\boldsymbol{U}_{h}}+\left\|\hat{\boldsymbol{v}}_{h}\right\|_{\boldsymbol{U}_{h}}\left\|\hat{\boldsymbol{w}}_{h}\right\|_{\boldsymbol{U}_{h}}+\chi \sum_{T \in \mathcal{T}_{h}} \nu_{2}^{-\frac{1}{2}} h_{T}^{\frac{1}{2}}\left\|\llbracket r_{T} \rrbracket_{\Gamma}\right\|_{T^{\Gamma}}\left\|\hat{\boldsymbol{w}}_{h}\right\|_{\boldsymbol{U}_{h}}
$$

where we used the boundedness of $a_{h}$ (cf Lemma 9). We now use the estimates

$$
\left\|\hat{\boldsymbol{w}}_{h}\right\|_{\boldsymbol{U}_{h}}^{2} \lesssim \nu_{1}\left|\boldsymbol{v}_{r_{h}}\right|_{H^{1}\left(\Omega_{1}\right)}^{2}+\nu_{2}\left|\boldsymbol{v}_{r_{h}}\right|_{H^{1}\left(\Omega_{2}\right)}^{2} \lesssim\left\|\nu^{-\frac{1}{2}} r_{h}\right\|_{L^{2}(\Omega)}^{2},
$$

and $\sum_{T \in \mathcal{T}_{h}}\left|\boldsymbol{E}_{i}\left(\boldsymbol{v}_{r_{h}}\right)\right|_{H^{1}\left(T^{\dagger}\right)}^{2} \lesssim\left|\boldsymbol{E}_{i}\left(\boldsymbol{v}_{r_{h}}\right)\right|_{H^{1}\left(\mathbb{R}^{d}\right)}^{2} \lesssim\left|\boldsymbol{v}_{r_{h}}\right|_{H^{1}\left(\Omega_{i}\right)}^{2} \lesssim\left\|\nu^{-\frac{1}{2}} r_{h}\right\|_{L^{2}(\Omega)}^{2}$ (see Lemma 6). With Young's inequality, the estimates on $\Psi_{1}$ and $\Psi_{2}$ give

$$
\sum_{T \in \mathcal{T}_{h}} \sum_{i \in\{1,2\}} \nu_{i}^{-1}\left\|r_{T^{i}}\right\|_{T^{i}}^{2} \lesssim S^{2}+\left\|\boldsymbol{v}_{h}\right\|_{\boldsymbol{U}_{h}}^{2}+\chi \sum_{T \in \mathcal{T}_{h}} \nu_{2}^{-1} h_{T}\left\|\llbracket r_{T} \rrbracket_{\Gamma}\right\|_{T^{\Gamma}}^{2}+\sum_{T \in \mathcal{T}_{h}} \sum_{i \in\{1,2\}} \nu_{i}^{-1} h_{T}^{2}\left\|\nabla r_{T^{i}}\right\|_{T^{i}}^{2} .
$$

Combined with (30) and (31), this bound implies that $\sum_{T \in \mathcal{T}_{h}} \sum_{i \in\{1,2\}} \nu_{i}^{-1}\left\|r_{T^{i}}\right\|_{T^{i}}^{2} \lesssim S^{2}+\chi^{-1} S\left\|\left(\hat{\boldsymbol{v}}_{h}, r_{h}\right)\right\|_{Y_{h}}$. Using again (30) and Young's inequality yields the inf-sup condition (29). Finally the inf-sup condition readily implies the well-posedness of (16) which amounts to a square linear system.

\subsection{Consistency and boundedness}

Let $(\boldsymbol{u}, p) \in H_{0}^{1}\left(\Omega ; \mathbb{R}^{d}\right) \times L_{*}^{2}(\Omega)$ be the solution to the exact problem (1). For all $i \in\{1,2\}$, let us set $\left(\boldsymbol{u}_{i}, p_{i}\right):=$ $\left(\boldsymbol{u}_{\mid \Omega_{i}}, p_{\mid \Omega_{i}}\right)$. For all $T \in \mathcal{T}_{h}$, let us define the discrete errors $\hat{\boldsymbol{e}}_{T}:=\hat{I}_{T}^{k}(\boldsymbol{u})-\hat{\boldsymbol{u}}_{T} \in \hat{\boldsymbol{U}}_{T}^{k}$ and $\eta_{T}:=J_{T}^{k}(p)-p_{T} \in P_{T}^{k}$, where $\left(\hat{\boldsymbol{u}}_{h}, p_{h}\right)$ is the solution to the discrete problem (16).

Lemma 11 (Consistency and boundedness). Assume that there is $t \in\left(\frac{1}{2}, k+1\right]$ such that $\left(\boldsymbol{u}_{i}, p_{i}\right) \in H^{t+1}\left(\Omega_{i} ; \mathbb{R}^{d}\right) \times$ $H^{t}\left(\Omega_{i}\right)$ for all $i \in\{1,2\}$. For all $y_{h}:=\left(\hat{\boldsymbol{v}}_{h}, q_{h}\right) \in Y_{h}^{k}:=\hat{\boldsymbol{U}}_{h 0}^{k} \times P_{h *}^{k}$, let us define

$$
\mathcal{F}\left(y_{h}\right):=\sum_{T \in \mathcal{T}_{h}} A_{T}\left(\left(\hat{\boldsymbol{e}}_{T}, \eta_{T}\right),\left(\hat{\boldsymbol{v}}_{T}, q_{T}\right)\right)
$$

Let $\boldsymbol{\delta}_{T^{i}}:=\boldsymbol{u}_{i}-I_{T^{i}}^{k+1}\left(\boldsymbol{u}_{i}\right), \mathbb{d}_{T^{i}}:=\nabla^{\mathrm{s}} \boldsymbol{u}_{i}-\mathbb{E}_{T^{i}}^{k}\left(\hat{I}_{T}^{k}(\boldsymbol{u})\right), d_{T^{i}}:=\nabla \cdot \boldsymbol{u}_{i}-D_{T^{i}}^{k}\left(\hat{I}_{T}^{k}(\boldsymbol{u})\right)$ and $\xi_{T^{i}}:=p_{i}-J_{T^{i}}^{k}(p)$, for all $T \in \mathcal{T}_{h}$. We have, for all $y_{h} \in Y_{h}^{k}$,

$$
\left|\mathcal{F}\left(y_{h}\right)\right| \lesssim\left\{\sum_{T \in \mathcal{T}_{h}}\left(\left\|\left(\mathbb{d}_{T}, \xi_{T}\right)\right\|_{* T}^{2}+\left\|\boldsymbol{\delta}_{T}\right\|_{\# T}^{2}+\sum_{i \in\{1,2\}} \nu_{i}\left\|d_{T^{i}}\right\|_{T^{i}}^{2}\right)\right\}^{\frac{1}{2}} \times\left\|y_{h}\right\|_{Y_{h}}
$$


where

$$
\begin{aligned}
\left\|\left(\mathbb{d}_{T}, \xi_{T}\right)\right\|_{* T}^{2}:= & \nu_{1} h_{T}\left\|\mathbb{d}_{T^{1}}\right\|_{T^{\Gamma}}^{2}+\sum_{i \in\{1,2\}}\left(\nu_{i}\left(\left\|\mathbb{d}_{T^{i}}\right\|_{T^{i}}^{2}+h_{T}\left\|\mathbb{d}_{T^{i}}\right\|_{(\partial T)^{i}}^{2}\right)\right. \\
& \left.+\nu_{i}^{-1}\left(\left\|\xi_{T^{i}}\right\|_{T^{i}}^{2}+h_{T}\left\|\xi_{T^{i}}\right\|_{(\partial T)^{i}}^{2}+h_{T}\left\|\xi_{T^{i}}\right\|_{T^{\Gamma}}^{2}\right)\right), \\
\left\|\boldsymbol{\delta}_{T}\right\|_{\# T}^{2}:= & \nu_{1} h_{T}^{-1}\left\|\boldsymbol{\delta}_{T^{1}}\right\|_{T^{\Gamma}}^{2}+\sum_{i \in\{1,2\}} \nu_{i}\left(h_{T}^{-1}\left\|\boldsymbol{\delta}_{T^{i}}\right\|_{(\partial T)^{i}}^{2}+h_{T}\left\|\nabla \boldsymbol{\delta}_{T^{i}}\right\|_{T^{\Gamma}}^{2}\right) .
\end{aligned}
$$

Proof. We observe that $\mathcal{F}\left(y_{h}\right)=\Psi_{1}+\Psi_{2}+\Psi_{3}$ with

$$
\begin{aligned}
\Psi_{1}:= & \sum_{T \in \mathcal{T}_{h}}\left(\sum_{i \in\{1,2\}} 2 \nu_{i}\left(\mathbb{E}_{T^{i}}^{k}\left(\hat{I}_{T}^{k}(\boldsymbol{u})\right), \mathbb{E}_{T^{i}}^{k}\left(\hat{\boldsymbol{v}}_{T}\right)\right)_{T^{i}}-\left(J_{T^{i}}^{k}(p), D_{T^{i}}^{k}\left(\hat{\boldsymbol{v}}_{T}\right)\right)_{T^{i}}+\left(\nabla \cdot\left(2 \nu_{i} \nabla^{\mathrm{s}} \boldsymbol{u}_{i}-p_{i} \mathbb{I}\right), \boldsymbol{v}_{T^{i}}\right)_{T^{i}}\right) \\
& -\left(\boldsymbol{g}_{N}, \boldsymbol{v}_{T^{2}}\right) T_{T^{\Gamma}}, \\
\Psi_{2}:= & \sum_{T \in \mathcal{T}_{h}} s_{T}\left(\hat{I}_{T}^{k}(\boldsymbol{u}), \hat{\boldsymbol{v}}_{T}\right)-\chi \nu_{2}^{-1} h_{T}\left(\llbracket \sigma\left(I_{T}^{k+1}(\boldsymbol{u}), J_{T}^{k}(p)\right) \rrbracket_{\Gamma} \boldsymbol{n}_{\Gamma}-\boldsymbol{g}_{N}, \llbracket \sigma\left(\boldsymbol{v}_{T},-q_{T}\right) \rrbracket_{\Gamma} \boldsymbol{n}_{\Gamma}\right)_{T^{\Gamma}}, \\
\Psi_{3}:= & \sum_{T \in \mathcal{T}_{h}} \sum_{i \in\{1,2\}}\left(D_{T^{i}}^{k}\left(\hat{I}_{T}^{k}(\boldsymbol{u})\right), q_{T^{i}}\right)_{T^{i}} .
\end{aligned}
$$

Using the definitions (8a)-(8b) of $\mathbb{E}_{T^{i}}^{k}\left(\hat{\boldsymbol{v}}_{T}\right)$, we infer that

$$
\begin{aligned}
& \sum_{T \in \mathcal{T}_{h}} \sum_{i \in\{1,2\}} 2 \nu_{i}\left(\mathbb{E}_{T^{i}}^{k}\left(\hat{I}_{T}^{k}(\boldsymbol{u})\right), \mathbb{E}_{T^{i}}^{k}\left(\hat{\boldsymbol{v}}_{T}\right)\right)_{T^{i}}=\sum_{T \in \mathcal{T}_{h}}\left\{-2 \nu_{1}\left(\mathbb{E}_{T^{1}}^{k}\left(\hat{I}_{T}^{k}(\boldsymbol{u})\right) \boldsymbol{n}_{\Gamma}, \llbracket \boldsymbol{v}_{T} \rrbracket_{\Gamma}\right)_{T^{\Gamma}}\right. \\
& \left.+\sum_{i \in\{1,2\}} 2 \nu_{i}\left(\left(\mathbb{E}_{T^{i}}^{k}\left(\hat{I}_{T}^{k}(\boldsymbol{u})\right), \nabla^{\mathrm{s}} \boldsymbol{v}_{T^{i}}\right)_{T^{i}}+\left(\mathbb{E}_{T^{i}}^{k}\left(\hat{I}_{T}^{k}(\boldsymbol{u})\right) \boldsymbol{n}_{T}, \boldsymbol{v}_{(\partial T)^{i}}-\boldsymbol{v}_{T^{i}}\right)_{(\partial T)^{i}}\right)\right\} .
\end{aligned}
$$

Similarly, using the definitions $(10 \mathrm{a})-(10 \mathrm{~b})$ of $D_{T^{i}}^{k}\left(\hat{\boldsymbol{v}}_{T}\right)$, we infer that

$$
\begin{aligned}
\sum_{T \in \mathcal{T}_{h}} \sum_{i \in\{1,2\}}\left(J_{T^{i}}^{k}(p), D_{T^{i}}^{k}\left(\hat{\boldsymbol{v}}_{T}\right)\right)_{T^{i}}= & \sum_{T \in \mathcal{T}_{h}}\left\{-\left(J_{T^{1}}^{k}(p) \boldsymbol{n}_{\Gamma}, \llbracket \boldsymbol{v}_{T} \rrbracket_{\Gamma}\right)_{T^{\Gamma}}\right. \\
& \left.+\sum_{i \in\{1,2\}}\left(\left(J_{T^{i}}^{k}(p), \nabla \cdot \boldsymbol{v}_{T^{i}}\right)_{T^{i}}+\left(J_{T^{i}}^{k}(p) \boldsymbol{n}_{T}, \boldsymbol{v}_{(\partial T)^{i}}-\boldsymbol{v}_{T^{i}}\right)_{(\partial T)^{i}}\right)\right\} .
\end{aligned}
$$

We integrate by parts on all the mesh cells. Moreover, since the trace of $2 \nu_{i} \nabla^{\mathrm{s}} \boldsymbol{u}_{i}-p_{i} \mathbb{I}$ over the mesh faces is well-defined owing to the regularity assumption made on the exact solution, we have $\sum_{T \in \mathcal{T}_{h}}\left(\left(-2 \nu_{i} \nabla^{\mathrm{s}} \boldsymbol{u}_{i}+\right.\right.$ $\left.\left.p_{i} \mathbb{I}\right) \boldsymbol{n}_{T}, \boldsymbol{v}_{(\partial T)^{i}}\right)_{(\partial T)^{i}}=0$. We infer that

$$
\begin{aligned}
\sum_{T \in \mathcal{T}_{h}} \sum_{i \in\{1,2\}} & \left(\nabla \cdot\left(2 \nu_{i} \nabla^{\mathrm{s}} \boldsymbol{u}_{i}-p_{i} \mathbb{I}\right), \boldsymbol{v}_{T^{i}}\right)_{T^{i}} \\
= & \sum_{T \in \mathcal{T}_{h}}\left\{\left(\left(2 \nu_{1} \nabla^{\mathrm{s}} \boldsymbol{u}_{1}-p_{1} \mathbb{I}\right) \boldsymbol{n}_{\Gamma}, \boldsymbol{v}_{T^{1}}\right)_{T^{\Gamma}}-\left(\left(2 \nu_{2} \nabla^{\mathrm{s}} \boldsymbol{u}_{2}-p_{2} \mathbb{I}\right) \boldsymbol{n}_{\Gamma}, \boldsymbol{v}_{T^{2}}\right)_{T^{\Gamma}}\right. \\
& \left.+\sum_{i \in\{1,2\}}\left(\left(-2 \nu_{i} \nabla^{\mathrm{s}} \boldsymbol{u}_{i}+p_{i} \mathbb{I}, \nabla^{\mathrm{s}} \boldsymbol{v}_{T^{i}}\right)_{T^{i}}+\left(\left(-2 \nu_{i} \nabla^{\mathrm{s}} \boldsymbol{u}_{i}+p_{i} \mathbb{I}\right) \boldsymbol{n}_{T}, \boldsymbol{v}_{(\partial T)^{i}}-\boldsymbol{v}_{T^{i}}\right)_{(\partial T)^{i}}\right)\right\} .
\end{aligned}
$$

Moreover we have

$$
\begin{aligned}
& \sum_{T \in \mathcal{T}_{h}}\left(\left(\left(2 \nu_{1} \nabla^{\mathrm{s}} \boldsymbol{u}_{1}-p_{1} \mathbb{I}\right) \boldsymbol{n}_{\Gamma}, \boldsymbol{v}_{T^{1}}\right)_{T^{\Gamma}}-\left(\left(2 \nu_{2} \nabla^{\mathrm{s}} \boldsymbol{u}_{2}-p_{2} \mathbb{I}\right) \boldsymbol{n}_{\Gamma}, \boldsymbol{v}_{T^{2}}\right)_{T^{\mathrm{T}}}\right) \\
& =\sum_{T \in \mathcal{T}_{h}}\left(\left(\llbracket \sigma(\boldsymbol{u}, p) \rrbracket_{\Gamma} \boldsymbol{n}_{\Gamma}, \boldsymbol{v}_{T^{2}}\right)_{T^{\Gamma}}+\left(\sigma\left(\boldsymbol{u}_{1}, p_{1}\right) \boldsymbol{n}_{\Gamma}, \llbracket \boldsymbol{v}_{T} \rrbracket_{\Gamma}\right)_{T^{\Gamma}}\right) .
\end{aligned}
$$

Putting the above four identities together we infer that

$$
\begin{aligned}
\Psi_{1}= & \sum_{T \in \mathcal{T}_{h}}\left\{\sum _ { i \in \{ 1 , 2 \} } \left(\left(-2 \nu_{i} \mathbb{d}_{T^{i}}, \nabla^{\mathrm{s}} \boldsymbol{v}_{T^{i}}\right)_{T^{i}}+\left(\xi_{T^{i}}, \nabla \cdot \boldsymbol{v}_{T^{i}}\right)_{T^{i}}\right.\right. \\
& \left.\left.+\left(\left(-2 \nu_{i} \mathrm{~d}_{T^{i}}+\xi_{T^{i}} \mathbb{I}\right) \boldsymbol{n}_{T}, \boldsymbol{v}_{(\partial T)^{i}}-\boldsymbol{v}_{T^{i}}\right)_{(\partial T)^{i}}\right)+\left(\left(2 \nu_{1} \mathbb{d}_{T^{1}}-\xi_{T^{1}} \mathbb{I}\right) \boldsymbol{n}_{\Gamma}, \llbracket \boldsymbol{v}_{T} \rrbracket_{\Gamma}\right)_{T^{\Gamma}}\right\} .
\end{aligned}
$$


Concerning $\Psi_{2}$, since $\Pi_{(\partial T)^{i}}^{k}$ is the $L^{2}$-orthogonal projection and $\llbracket \boldsymbol{u} \rrbracket_{\Gamma}=\mathbf{0}$ on $T^{\Gamma}$, we have

$$
\begin{aligned}
\Psi_{2}= & \sum_{T \in \mathcal{T}_{h}}\left\{-\nu_{1} h_{T}^{-1}\left(\llbracket \boldsymbol{\delta}_{T} \rrbracket_{\Gamma}, \llbracket \boldsymbol{v}_{T} \rrbracket_{\Gamma}\right)_{T^{\Gamma}}+\chi \nu_{2}^{-1} h_{T}\left(\llbracket \sigma\left(\boldsymbol{\delta}_{T}, \xi_{T}\right) \rrbracket_{\Gamma} \boldsymbol{n}_{\Gamma}, \llbracket \sigma\left(\boldsymbol{v}_{T},-q_{T}\right) \rrbracket_{\Gamma} \boldsymbol{n}_{\Gamma}\right)_{T^{\Gamma}}\right. \\
& \left.+\sum_{i \in\{1,2\}} \nu_{i} h_{T}^{-1}\left(\boldsymbol{\delta}_{T^{i}}, \boldsymbol{v}_{(\partial T)^{i}}-\Pi_{(\partial T)^{i}}^{k}\left(\boldsymbol{v}_{T^{i}}\right)\right)_{(\partial T)^{i}}\right\} .
\end{aligned}
$$

Finally, concerning $\Psi_{3}$, we have $\nabla \cdot \boldsymbol{u}=0$, which implies that

$$
\Psi_{3}=-\sum_{T \in \mathcal{T}_{h}} \sum_{i \in\{1,2\}}\left(d_{T^{i}}, q_{T^{i}}\right)_{T^{i}}
$$

The final estimate follows from the Cauchy-Schwarz inequality.

\subsection{Error estimate}

Let $(\boldsymbol{u}, p) \in H_{0}^{1}\left(\Omega ; \mathbb{R}^{d}\right) \times L_{*}^{2}(\Omega)$ be the solution to the exact problem (1) and $\left(\hat{\boldsymbol{u}}_{h}, p_{h}\right)$ be the solution to the discrete problem (16).

Theorem 12. Let $k \geq 1$. Assume that there is $t \in\left(\frac{1}{2}, k+1\right]$ such that $\left(\boldsymbol{u}_{i}, p_{i}\right) \in H^{t+1}\left(\Omega_{i} ; \mathbb{R}^{d}\right) \times H^{t}\left(\Omega_{i}\right)$ for all $i \in\{1,2\}$. There is $C$, scaling linearly with $\chi^{-1}$, such that the following holds true:

$$
\begin{aligned}
& \sum_{T \in \mathcal{T}_{h}} \sum_{i \in\{1,2\}}\left(\nu_{i}\left\|\nabla^{\mathrm{s}}\left(\boldsymbol{u}_{i}-\boldsymbol{u}_{T^{i}}\right)\right\|_{T^{i}}^{2}+\nu_{i}^{-1}\left\|p_{i}-p_{T^{i}}\right\|_{T^{i}}^{2}\right) \leq C \times \\
& \sum_{T \in \mathcal{T}_{h}}\left\{\left\|\left(\mathbb{d}_{T}, \xi_{T}\right)\right\|_{* T}^{2}+\left\|\boldsymbol{\delta}_{T}\right\|_{\# T}^{2}+\sum_{i \in\{1,2\}}\left(\nu_{i}\left\|\nabla^{\mathrm{s}}\left(\boldsymbol{u}_{i}-I_{T^{i}}^{k+1}(\boldsymbol{u})\right)\right\|_{T^{i}}^{2}+\nu_{i}^{-1}\left\|p_{i}-J_{T^{i}}^{k}(p)\right\|_{T^{i}}^{2}+\nu_{i}\left\|d_{T^{i}}\right\|_{T^{i}}^{2}\right)\right\} .
\end{aligned}
$$

Moreover, we have

$$
\sum_{T \in \mathcal{T}_{h}} \sum_{i \in\{1,2\}}\left(\nu_{i}\left\|\nabla^{\mathrm{s}}\left(\boldsymbol{u}_{i}-\boldsymbol{u}_{T^{i}}\right)\right\|_{T^{i}}^{2}+\nu_{i}^{-1}\left\|p_{i}-p_{T^{i}}\right\|_{T^{i}}^{2}\right) \lesssim h^{2 t} \sum_{i \in\{1,2\}}\left(\nu_{i}|\boldsymbol{u}|_{H^{t+1}\left(\Omega_{i}\right)}^{2}+\nu_{i}^{-1}|p|_{H^{t}\left(\Omega_{i}\right)}^{2}\right) .
$$

Proof. Owing to the triangle inequality, we have

$$
\begin{aligned}
& \sum_{T \in \mathcal{T}_{h}} \sum_{i \in\{1,2\}}\left(\nu_{i}\left\|\nabla^{\mathrm{s}}\left(\boldsymbol{u}_{i}-\boldsymbol{u}_{T^{i}}\right)\right\|_{T^{i}}^{2}+\nu_{i}^{-1}\left\|p_{i}-p_{T^{i}}\right\|_{T^{i}}^{2}\right) \\
& \leq 2 \sum_{T \in \mathcal{T}_{h}} \sum_{i \in\{1,2\}}\left(\nu_{i}\left\|\nabla^{\mathrm{s}}\left(\boldsymbol{u}_{i}-I_{T^{i}}^{k+1}(\boldsymbol{u})\right)\right\|_{T^{i}}^{2}+\nu_{i}^{-1}\left\|p_{i}-J_{T^{i}}^{k}(p)\right\|_{T^{i}}^{2}\right)+2\left\|\left(\hat{\boldsymbol{e}}_{h}, \eta_{h}\right)\right\|_{Y_{h}}^{2} .
\end{aligned}
$$

According to Lemma 10, we have

$$
\beta\left\|\left(\hat{\boldsymbol{e}}_{h}, \eta_{h}\right)\right\|_{Y_{h}} \leq \sup _{y_{h} \in Y_{h}^{k} \backslash\{0\}} \frac{A_{h}\left(\left(\hat{\boldsymbol{e}}_{h}, \eta_{h}\right), y_{h}\right)}{\left\|y_{h}\right\|_{Y_{h}}}=\sup _{y_{h} \in Y_{h}^{k} \backslash\{0\}} \frac{\mathcal{F}\left(y_{h}\right)}{\left\|y_{h}\right\|_{Y_{h}}} .
$$

Moreover, according to Lemma 11, we have

$$
\left|\mathcal{F}\left(y_{h}\right)\right| \lesssim\left\{\sum_{T \in \mathcal{T}_{h}}\left(\left\|\left(\mathbb{d}_{T}, \xi_{T}\right)\right\|_{* T}^{2}+\left\|\boldsymbol{\delta}_{T}\right\|_{\# T}^{2}+\sum_{i \in\{1,2\}} \nu_{i}\left\|d_{T^{i}}\right\|_{T^{i}}^{2}\right)\right\}^{\frac{1}{2}}\left\|y_{h}\right\|_{Y_{h}} .
$$

Combining these bounds proves the first error estimate. Finally invoking the approximation properties of $\hat{I}_{T}^{k}(\boldsymbol{v})$ (see Lemmas 6 and 7), we obtain the second estimate.

\section{$5 \quad$ Numerical simulations}

In this section, the global domain is the unit square $\Omega:=(0,1)^{2}$, and the interface is a circle of center $(0.5,0.5)$ and radius $R=1 / 3$. The subdomain $\Omega_{2}$ where the viscosity is higher lies inside the circular interface, and the subdomain $\Omega_{1}$ lies outside. We consider Cartesian meshes of mesh size $h:=\sqrt{2} / N$, where $N=8,16,32$ and 64 . We consider that a cell $T \in \mathcal{T}_{h}^{\Gamma}$ has a small cut if $\left|T^{i}\right| \leq 0.3|T|$ for some $i \in\{1,2\}$, where $\left|T^{i}\right|$ and $|T|$ are the volumes of $T^{i}$ and $T$, respectively. Such small cut cells are agglomerated following the procedure described in 

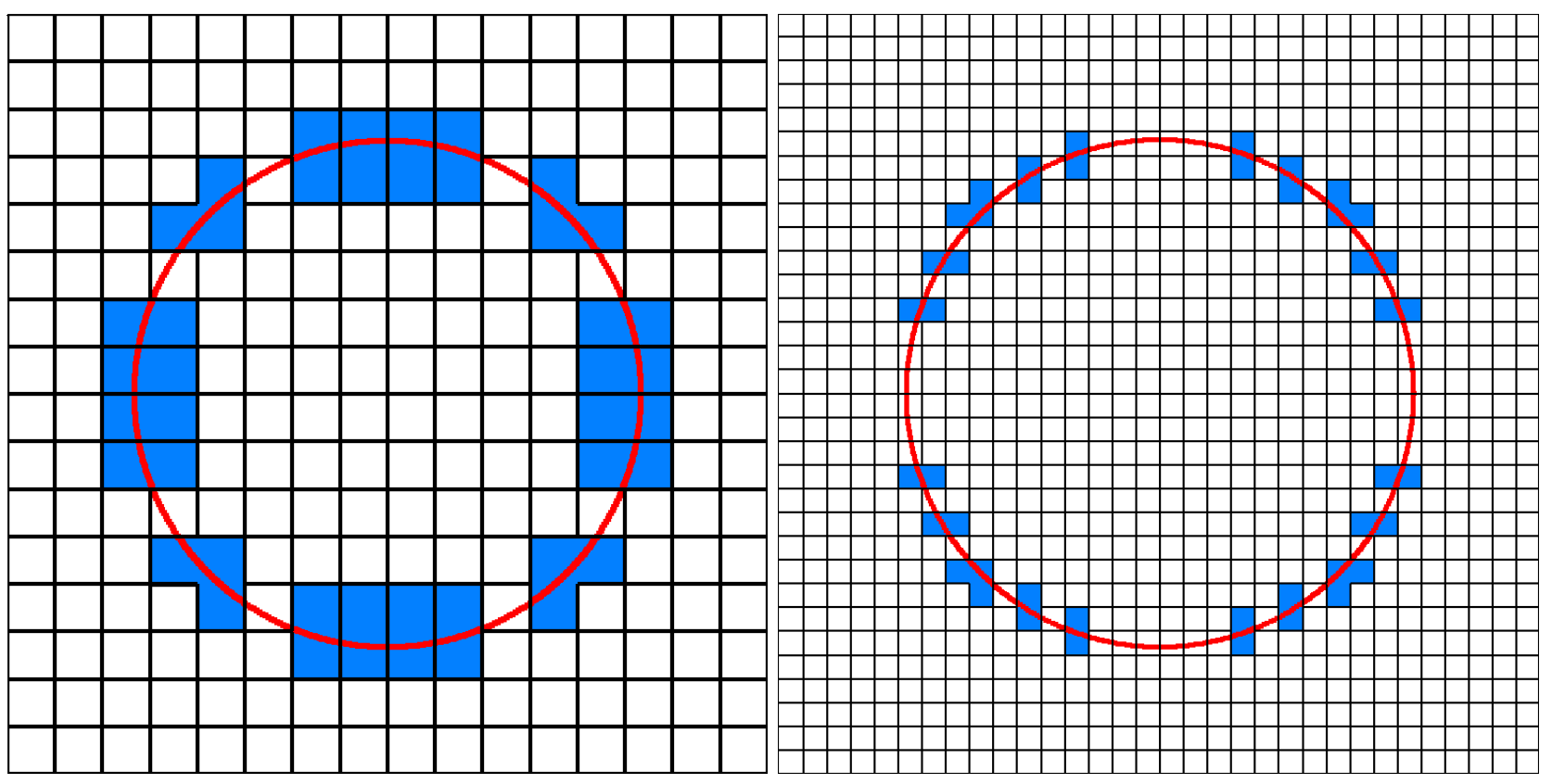

Figure 1: The domain, the mesh and the interface for $h=\sqrt{2} / 16$ (left) and $h=\sqrt{2} / 32$ (right). The agglomerated cells are highlighted.

Algorithm 1 of [9]. The domain, the interface and the meshes (for $h=\sqrt{2} / 16$ and $h=\sqrt{2} / 32$ ) are presented in Figure 1.

The integration over the curved interface is done following the method proposed in Section 4.2 of [9]. In every cut cell $T \in \mathcal{T}_{h}^{\Gamma}$, the interface is represented by $2^{n_{\text {int }}}$ segments, where $n_{\text {int }}$ is a positive integer. The numerical integration over the interface is the sum of the integrations over all the segments. Choosing $n_{\text {int }}$ higher means that we approximate the interface in a better way but makes the method more expensive. In a similar way, the integrations over the cut cells are carried out by dividing the cut cells into several triangles. The implementation is realized using the DiSk++ library available from https://github.com/wareHHOuse (see also [15]).

We consider two test cases: a test case with a pressure jump across the interface and a test case with a contrast in the viscosity coefficient. The exact solution is denoted $(\boldsymbol{u}, p)$. We report the errors $\left(\sum_{i \in\{1,2\}} \sum_{T \in \mathcal{T}_{h}} \nu_{i} \| \nabla^{\mathrm{s}}(\boldsymbol{u}-\right.$ $\left.\left.\boldsymbol{u}_{T^{i}}\right) \|_{T^{i}}^{2}\right)^{\frac{1}{2}}$ for the velocity and $\left(\sum_{i \in\{1,2\}} \sum_{T \in \mathcal{T}_{h}} \nu_{i}^{-1}\left\|p-p_{T^{i}}\right\|_{T^{i}}^{2}\right)^{\frac{1}{2}}$ for the pressure.

Even if the theoretical analysis of the unfitted HHO method requires $\chi>0$, our numerical tests indicate that the method works perfectly well with $\chi=0$. All the numerical simulations are run with $\chi=0$. Moreover, although the theoretical analysis requires $k \geq 1$ for the polynomial degree, we also include in our numerical tests the lowest-order case $k=0$.

\subsection{Test case with pressure jump}

We consider here a test case where the velocity is null in the whole domain, and the pressure is discontinuous across the circular interface. We have

$$
\begin{array}{llrl}
\boldsymbol{u}(x, y) & :=(0,0) & \forall(x, y) \in \Omega, \\
p(x, y):=-\pi R K & \forall(x, y) \in \Omega_{1}, \\
p(x, y):=\frac{K}{R}-\pi R K & \forall(x, y) \in \Omega_{2},
\end{array}
$$

where the pressure jump is proportional to the curvature of the interface since we have $\llbracket \sigma(u, p) \boldsymbol{n}_{\Gamma} \rrbracket_{\Gamma}=-\frac{K}{R}$. Moreover, $\nu_{1}=\nu_{2}=1$. This test case was proposed in [22]. Note that the mean pressure is null. In our tests we consider $K:=0.05$.

A plot of the discrete pressure is shown in the left panel of Figure 2, whereas the pressure errors are reported in the right panel of Figure 2. A more comprehensive convergence overview is provided in Tables 1 and 2 for the velocity and the pressure respectively. We observe that the exact solution is a constant function in each subdomain. Theorem 12 then implies that the errors vanish in the absence of geometric errors on the representation of the interface. Therefore we expect the errors to tend to zero when $n_{\text {int }}$ is increased. This is well reflected in our numerical results since we observe that the error diminishes when $n_{\text {int }}$ is increased (see 

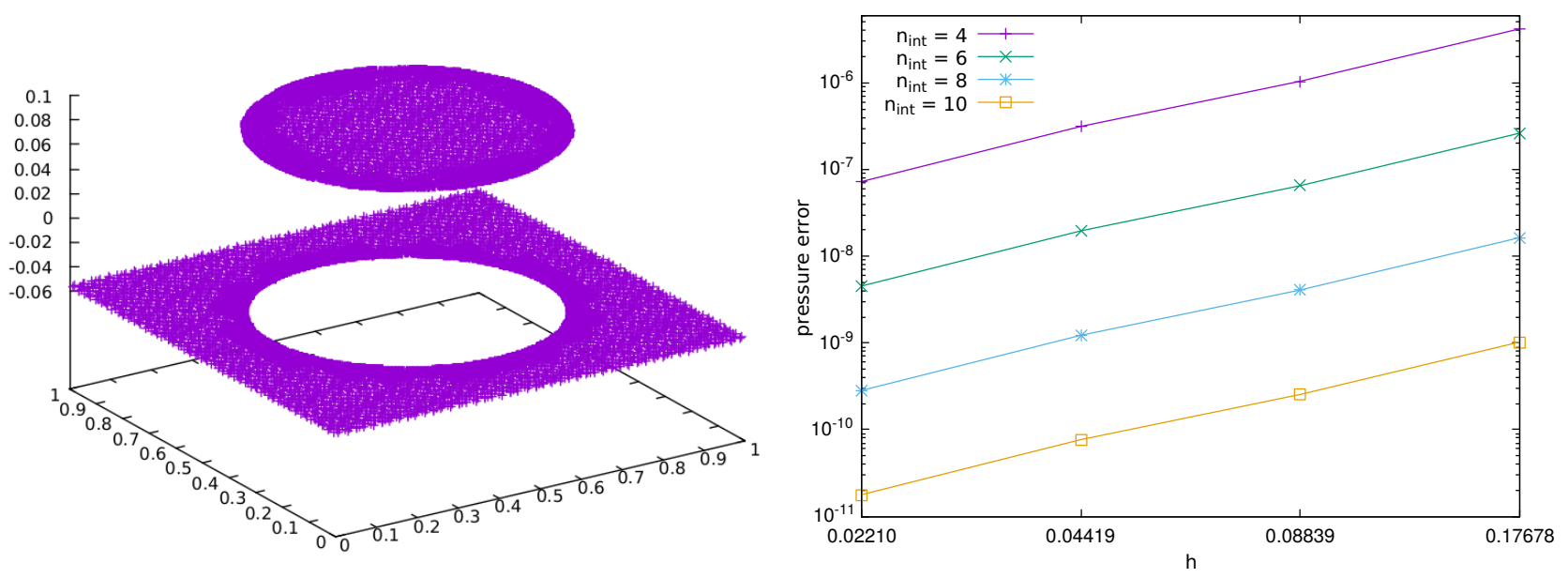

Figure 2: Test case with pressure jump. Left: discrete pressure for $h=\sqrt{2} / 16$ and $k=1$. Right: pressure error for $k=1$.

\begin{tabular}{|c|r|c|c|c|c|}
\hline & \multicolumn{1}{|c|}{$h$} & $\sqrt{2} / 8$ & $\sqrt{2} / 16$ & $\sqrt{2} / 32$ & $\sqrt{2} / 64$ \\
\hline \multirow{5}{*}{$n_{\text {int }}=4$} & $k=0$ & $2.42 \mathrm{e}-05$ & $1.03 \mathrm{e}-05$ & $4.93 \mathrm{e}-06$ & $1.63 \mathrm{e}-06$ \\
& 1 & $4.97 \mathrm{e}-09$ & $1.11 \mathrm{e}-09$ & $3.20 \mathrm{e}-10$ & $4.20 \mathrm{e}-11$ \\
& 2 & $2.59 \mathrm{e}-12$ & $3.01 \mathrm{e}-13$ & $7.64 \mathrm{e}-14$ & $8.85 \mathrm{e}-13$ \\
& 3 & $6.32 \mathrm{e}-15$ & $9.25 \mathrm{e}-15$ & $7.29 \mathrm{e}-14$ & $6.63 \mathrm{e}-13$ \\
\hline \multirow{5}{*}{$n_{\text {int }}=6$} & $k=0$ & $1.51 \mathrm{e}-06$ & $6.47 \mathrm{e}-07$ & $3.08 \mathrm{e}-07$ & $1.02 \mathrm{e}-07$ \\
& 1 & $1.94 \mathrm{e}-11$ & $4.33 \mathrm{e}-12$ & $1.25 \mathrm{e}-12$ & $1.40 \mathrm{e}-12$ \\
& 2 & $1.85 \mathrm{e}-15$ & $5.16 \mathrm{e}-15$ & $5.61 \mathrm{e}-14$ & $7.00 \mathrm{e}-13$ \\
& 3 & $5.27 \mathrm{e}-15$ & $8.79 \mathrm{e}-15$ & $8.38 \mathrm{e}-14$ & $6.87 \mathrm{e}-13$ \\
\hline \multirow{5}{*}{$n_{\text {int }}=8$} & $k=0$ & $9.45 \mathrm{e}-08$ & $4.04 \mathrm{e}-08$ & $1.92 \mathrm{e}-08$ & $6.38 \mathrm{e}-09$ \\
& 1 & $7.57 \mathrm{e}-14$ & $1.78 \mathrm{e}-14$ & $3.69 \mathrm{e}-14$ & $7.78 \mathrm{e}-13$ \\
& 2 & $2.55 \mathrm{e}-15$ & $5.12 \mathrm{e}-15$ & $5.76 \mathrm{e}-14$ & $7.69 \mathrm{e}-13$ \\
& 3 & $7.63 \mathrm{e}-15$ & $1.11 \mathrm{e}-14$ & $7.82 \mathrm{e}-14$ & $5.54 \mathrm{e}-13$ \\
\hline \multirow{5}{*}{$n_{\text {int }}=10$} & $k=0$ & $5.91 \mathrm{e}-09$ & $2.53 \mathrm{e}-09$ & $1.20 \mathrm{e}-09$ & $3.99 \mathrm{e}-10$ \\
& 1 & $1.29 \mathrm{e}-15$ & $7.98 \mathrm{e}-15$ & $5.18 \mathrm{e}-14$ & $7.34 \mathrm{e}-13$ \\
& 2 & $3.72 \mathrm{e}-15$ & $4.78 \mathrm{e}-15$ & $5.54 \mathrm{e}-14$ & $8.42 \mathrm{e}-13$ \\
& 3 & $1.42 \mathrm{e}-14$ & $1.60 \mathrm{e}-14$ & $9.54 \mathrm{e}-14$ & $5.31 \mathrm{e}-13$ \\
\hline
\end{tabular}

Table 1: Test case with pressure jump: velocity errors for various meshes (parameter $h$ ), polynomial degrees (parameter $k$ ), and geometric resolutions of the interface (parameter $n_{\text {int }}$ ).

Figure 2 and Tables 1,2). As can be expected for very small errors, increasing $n_{\text {int }}$ is no longer beneficial. We expect that it is due to the fact that the number of operations increases with $n_{\text {int }}$ and thus the rounding in floating point arithmetics can no longer be neglected (the unknowns are represented with double precision).

On the same token, we expect that increasing the polynomial degree $k$ will not improve the numerical results since the error is due to the geometric error in the representation of the interface. As expected we can see in Table 2 that the pressure error is the same for $k=1,2,3$. It is however larger for $k=0$. An interesting result is that we observe numerically the convergence of the scheme even for the case $k=0$, which is not covered by the present analysis, although the errors are somewhat larger than for $k \geq 1$. Thus we conjecture that the convergence of the scheme can also be obtained for $k=0$ but with less favorable constants. A similar behavior has already been observed in Section 4.5.2 of [32] in the context of elasticity, where the author reported that the method remains convergent for $k=0$ provided the cells have at least $2 d$ faces, i.e. quadrangles in $2 d$ and hexahedra in $3 d$. We concur with these numerical observations for the present Stokes interface problem using quadrangles in $2 d$. These numerical observations still require a theoretical justification. Notice that a provably well-posed scheme for $k=0$ is devised in [7] by penalizing the jumps of the cell unknowns thereby leading to a global coupling of these unknowns as well. 


\begin{tabular}{|c|r|c|c|c|c|}
\hline & \multicolumn{1}{|c|}{$h$} & $\sqrt{2} / 8$ & $\sqrt{2} / 16$ & $\sqrt{2} / 32$ & $\sqrt{2} / 64$ \\
\hline \multirow{5}{*}{$n_{\text {int }}=4$} & $k=0$ & $6.11 \mathrm{e}-06$ & $1.57 \mathrm{e}-06$ & $6.32 \mathrm{e}-07$ & $1.76 \mathrm{e}-07$ \\
& 1 & $4.19 \mathrm{e}-06$ & $1.04 \mathrm{e}-06$ & $3.13 \mathrm{e}-07$ & $7.24 \mathrm{e}-08$ \\
& 2 & $4.19 \mathrm{e}-06$ & $1.04 \mathrm{e}-06$ & $3.13 \mathrm{e}-07$ & $7.24 \mathrm{e}-08$ \\
& 3 & $4.19 \mathrm{e}-06$ & $1.04 \mathrm{e}-06$ & $3.13 \mathrm{e}-07$ & $7.24 \mathrm{e}-08$ \\
\hline \multirow{5}{*}{$n_{\text {int }}=6$} & $k=0$ & $3.82 \mathrm{e}-07$ & $9.81 \mathrm{e}-08$ & $3.95 \mathrm{e}-08$ & $1.10 \mathrm{e}-08$ \\
& 1 & $2.62 \mathrm{e}-07$ & $6.51 \mathrm{e}-08$ & $1.96 \mathrm{e}-08$ & $4.53 \mathrm{e}-09$ \\
& 2 & $2.62 \mathrm{e}-07$ & $6.51 \mathrm{e}-08$ & $1.96 \mathrm{e}-08$ & $4.53 \mathrm{e}-09$ \\
& 3 & $2.62 \mathrm{e}-07$ & $6.51 \mathrm{e}-08$ & $1.96 \mathrm{e}-08$ & $4.53 \mathrm{e}-09$ \\
\hline \multirow{5}{*}{$n_{\text {int }}=8$} & $k=0$ & $2.39 \mathrm{e}-08$ & $6.13 \mathrm{e}-09$ & $2.47 \mathrm{e}-09$ & $6.88 \mathrm{e}-10$ \\
& 1 & $1.64 \mathrm{e}-08$ & $4.07 \mathrm{e}-09$ & $1.22 \mathrm{e}-09$ & $2.83 \mathrm{e}-10$ \\
& 2 & $1.64 \mathrm{e}-08$ & $4.07 \mathrm{e}-09$ & $1.22 \mathrm{e}-09$ & $2.83 \mathrm{e}-10$ \\
& 3 & $1.64 \mathrm{e}-08$ & $4.07 \mathrm{e}-09$ & $1.22 \mathrm{e}-09$ & $2.83 \mathrm{e}-10$ \\
\hline \multirow{5}{*}{$n_{\text {int }}=10$} & $k=0$ & $1.49 \mathrm{e}-09$ & $3.83 \mathrm{e}-10$ & $1.54 \mathrm{e}-10$ & $4.30 \mathrm{e}-11$ \\
& 1 & $1.02 \mathrm{e}-09$ & $2.54 \mathrm{e}-10$ & $7.65 \mathrm{e}-11$ & $1.77 \mathrm{e}-11$ \\
& 2 & $1.02 \mathrm{e}-09$ & $2.54 \mathrm{e}-10$ & $7.65 \mathrm{e}-11$ & $1.78 \mathrm{e}-11$ \\
& 3 & $1.02 \mathrm{e}-09$ & $2.54 \mathrm{e}-10$ & $7.65 \mathrm{e}-11$ & $1.77 \mathrm{e}-11$ \\
\hline
\end{tabular}

Table 2: Test case with pressure jump: pressure errors for various meshes (parameter $h$ ), polynomial degrees (parameter $k$ ), and geometric resolutions of the interface (parameter $n_{\text {int }}$ ).

\subsection{Test case with contrasted viscosity}

In this section, we consider the following test case:

$$
\begin{aligned}
& \boldsymbol{u}(r, \theta):=\tilde{u}(r)(\sin \theta,-\cos \theta), \\
& p(r):=r^{4}-\frac{7}{180},
\end{aligned}
$$

where $r^{2}:=(x-0.5)^{2}+(y-0.5)^{2}, x-0.5:=r \sin \theta, y-0.5:=r \sin \theta$ and

$$
\tilde{u}(r)= \begin{cases}\frac{r^{6}}{\nu_{2}} & \text { if } r<R \\ \frac{r^{6}-R^{6}}{\nu_{1}}+\frac{R^{6}}{\nu_{2}} & \text { otherwise. }\end{cases}
$$

Notice that the pressure has zero mean-value. Moreover we have $\boldsymbol{g}_{N}=\left(1-\frac{\nu_{1}}{\nu_{2}}\right) r^{5}\left(\begin{array}{c}-\sin \theta \\ \cos \theta\end{array}\right)$ and

$$
\boldsymbol{f}=4 r^{3}\left(\begin{array}{l}
\cos \theta \\
\sin \theta
\end{array}\right)+\left(\begin{array}{c}
-\sin \theta \\
\cos \theta
\end{array}\right) \times\left\{\begin{array}{lr}
35 r^{4} & \text { if } r \leq R \\
35 r^{4}+\frac{\nu_{2}-\nu_{1}}{\nu_{2}} \frac{R^{6}}{r^{2}} & \text { otherwise. }
\end{array}\right.
$$

The interesting feature of this test case is that $\sum_{i \in\{1,2\}}\left(\nu_{i}|\boldsymbol{u}|_{H^{k+2}\left(\Omega_{i}\right)}^{2}+\nu_{i}^{-1}|p|_{H^{k+1}\left(\Omega_{i}\right)}^{2}\right)$ remains bounded for $\nu_{1}=1$ when $\nu_{2}$ becomes large. We can then study the variation of the error when the viscosity contrast, as measured by the ratio $\nu_{2} / \nu_{1}$, increases. We expect from the theoretical analysis that the error remains bounded for high contrasts. The elevation for the velocity magnitude and the pressure is presented in Figure 3 .

The errors (velocity in $H^{1}$-seminorm, pressure in $L^{2}$-norm) are reported in Figures 4,5 and 6 . In Figure 4 we present the errors with respect to the mesh size $h$ for various polynomial degrees. We recover the convergence rates stated in Theorem 12. In Figure 5, we report the errors with respect to the viscosity contrast $\frac{\nu_{2}}{\nu_{1}}$ for various polynomial degrees. These results confirm that the method is robust with respect to the viscosity contrast $\nu_{2} / \nu_{1}$ (as soon as the geometric representation of the interface is fine enough). The error is somewhat larger for $\nu_{2}=10^{6}$ when $k=3$. In order to study this phenomenon, we draw in Figure 6 the error with respect to the geometric resolution parameter $n_{\text {int }}$ for various viscosity contrasts. We observe that the error tends to the same value for $\nu_{2}=1,10^{2}, 10^{4}$ but not for $\nu_{2}=10^{6}$. Since the norm of the error scales as $\nu_{2}^{\frac{1}{2}}$, this behavior can be interpreted as a consequence of multiplying rounding errors by a large factor.

\section{Conclusions}

In this work we designed and analyzed an unfitted HHO method for the Stokes interface problem (with symmetric gradients). Error estimates have been established for polynomial degrees $k \geq 1$ and for a penalty parameter $\chi>0$ that is small enough. The same results can be established for the formulation with full gradients (in this case $\sigma(\boldsymbol{u}, p):=\nu \nabla \boldsymbol{u}-p \mathbb{I})$, the only difference being that we do not need the assumption $k \geq 1$ at the 

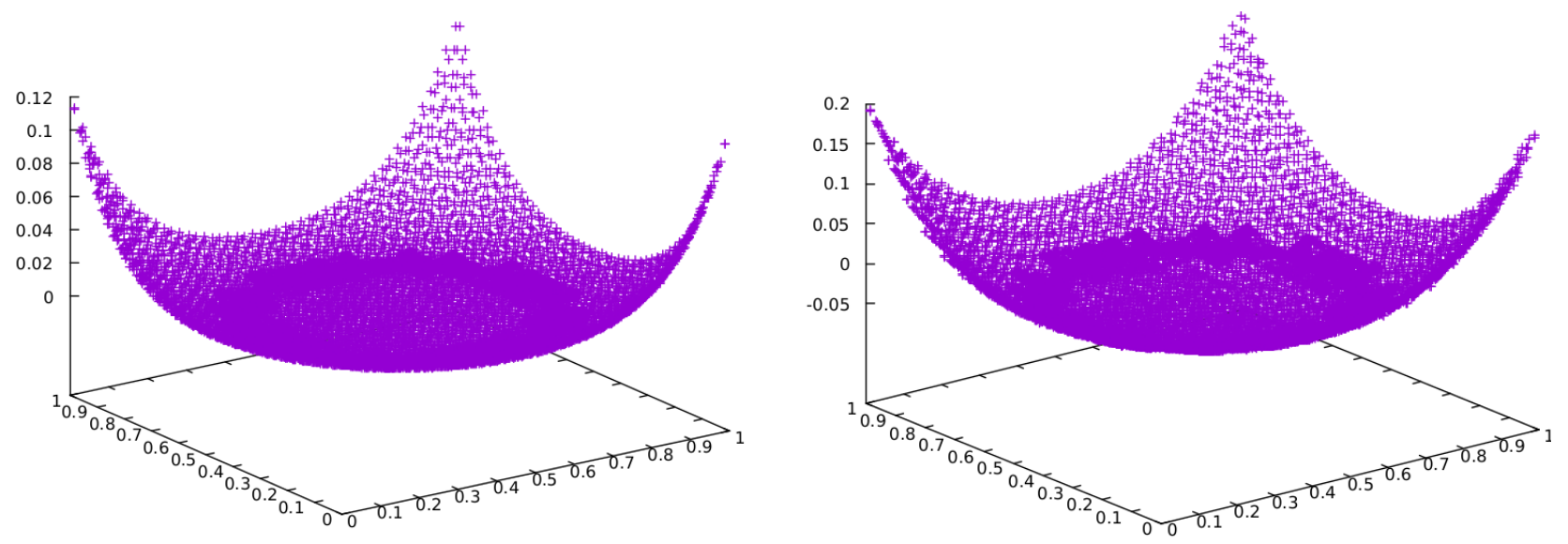

Figure 3: Test case with contrasted viscosity $\left(\nu_{1}=1.0, \nu_{2}=10^{4}\right), n_{\text {int }}=4, k=1, h=\sqrt{2} / 16$. Elevation for the velocity magnitude (left) and the pressure (right).
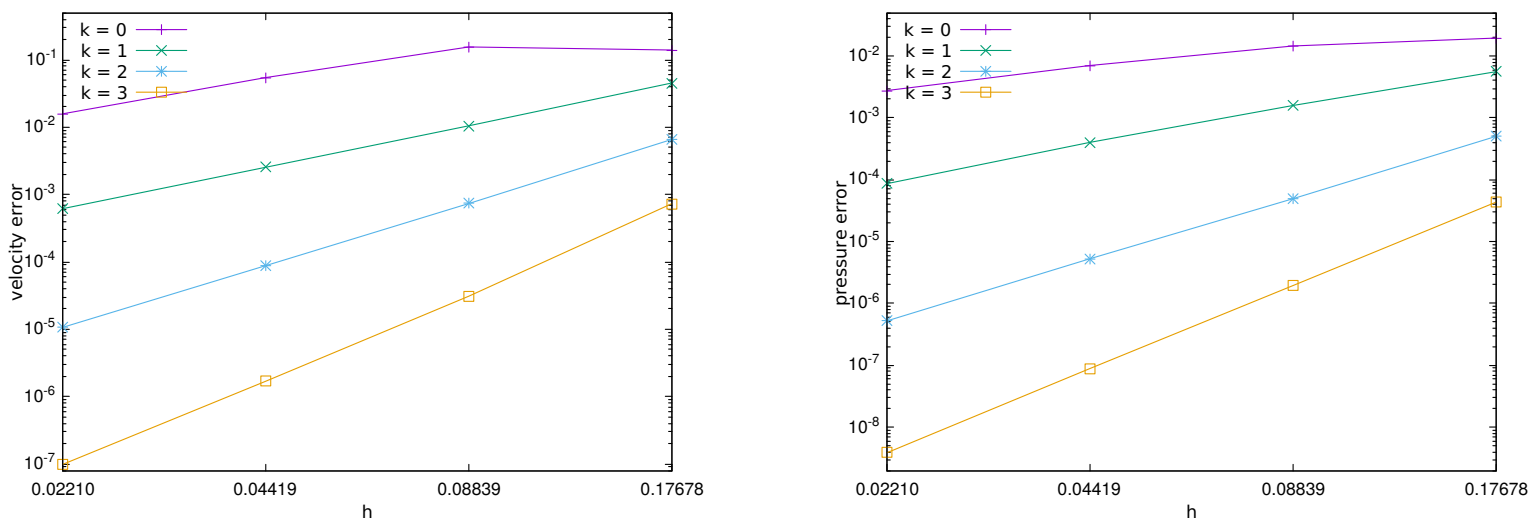

Figure 4: Test case with contrasted viscosity $\left(\nu_{1}=1.0, \nu_{2}=10^{4}\right), n_{\text {int }}=10$. Errors (left: velocity in $H^{1}$ seminorm; right: pressure in $L^{2}$-norm) as a function of the mesh size for various polynomial degrees.

theoretical level since Korn's inequality is not used. Neglecting geometric errors in the representation of the interface and quadrature errors in the cut and uncut cells, we have proved a priori error estimates that are optimally convergent with respect to the mesh size and robust with respect to the viscosity contrast.

Finally the present integration method relies on a discretization of the interface with a step much finer than the size of the cells. This enables us to recover in numerical simulations the a priori convergence rates. However, for very high contrasts or for very fine meshes this approach is not sufficient to compute a very precise solution, and calls for the development of another integration method. As a recent example we can mention the isoparametric representation of the interface considered in [30].

\section{References}

[1] S. Adjerid, N. Chaabane, T. Lin, and P. Yue. An immersed discontinuous finite element method for the Stokes problem with a moving interface. J. Comput. Appl. Math., 362:540-559, 2019.

[2] J. W. Barrett and C. M. Elliott. A finite-element method for solving elliptic equations with Neumann data on a curved boundary using unfitted meshes. IMA J. Numer. Anal., 4(3):309-325, 1984.

[3] J. W. Barrett and C. M. Elliott. Fitted and unfitted finite-element methods for elliptic equations with smooth interfaces. IMA J. Numer. Anal., 7(3):283-300, 1987.

[4] R. Becker, E. Burman, and P. Hansbo. A Nitsche extended finite element method for incompressible elasticity with discontinuous modulus of elasticity. Comput. Methods Appl. Mech. Engrg., 198(41-44):3352$3360,2009$.

[5] L. Botti, D. A. Di Pietro, and J. Droniou. A Hybrid High-Order discretisation of the Brinkman problem robust in the Darcy and Stokes limits. Comput. Methods Appl. Mech. Engrg., 341:278-310, 2018. 

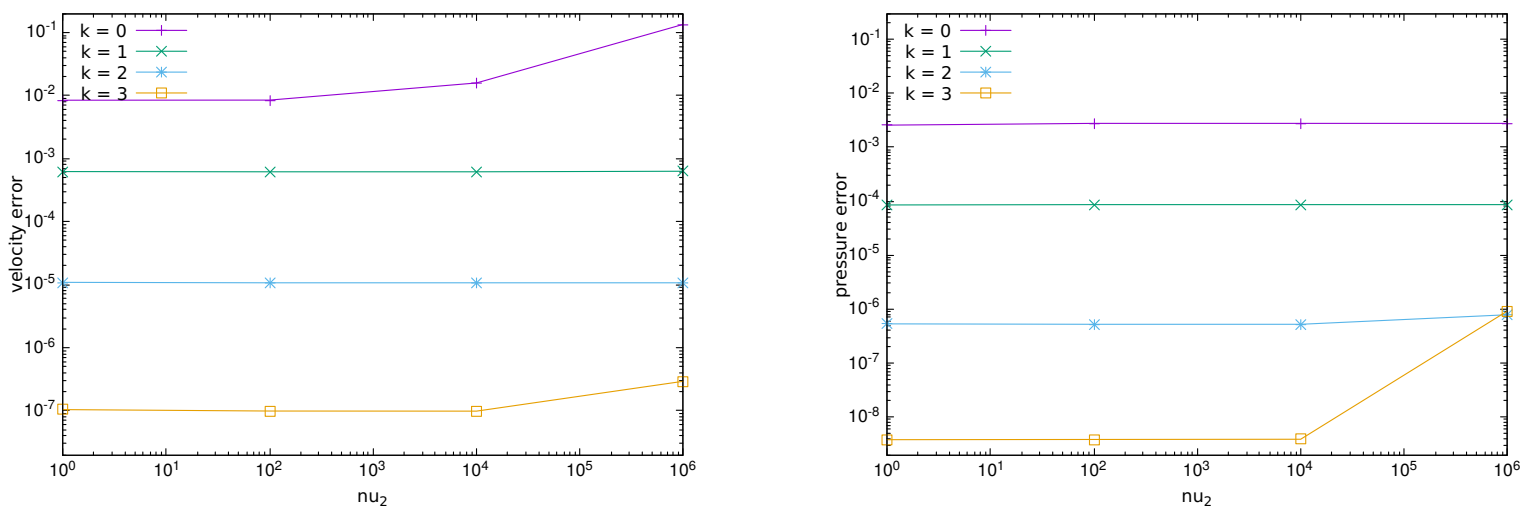

Figure 5: Test case with contrasted viscosity $\left(\nu_{1}=1.0\right), n_{\text {int }}=10$, and $h=\sqrt{2} / 64$. Errors (left: velocity in $H^{1}$-seminorm; right: pressure in $L^{2}$-norm) as a function of the viscosity contrast for various polynomial degrees.
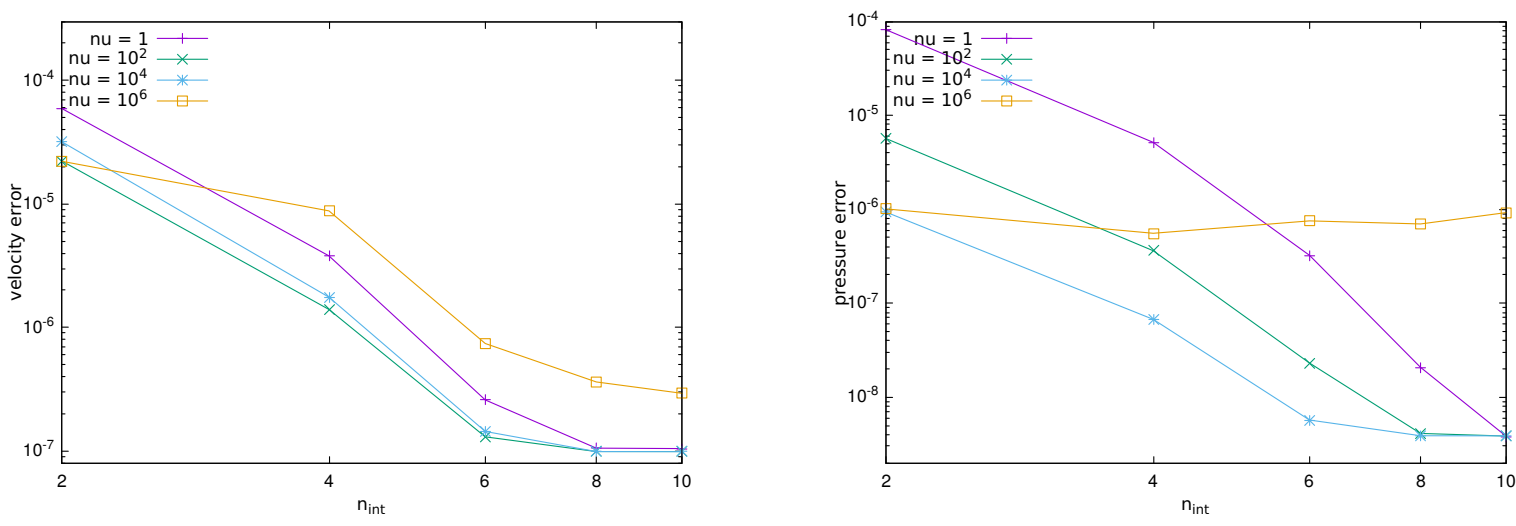

Figure 6: Test case with contrasted viscosity $\left(\nu_{1}=1.0\right), h=\sqrt{2} / 64$, and $k=3$. Errors (left: velocity in $H^{1}$-seminorm; right: pressure in $L^{2}$-norm) as a function of the geometric resolution of the interface for various viscosity contrasts.

[6] L. Botti, D. A. Di Pietro, and J. Droniou. A hybrid high-order method for the incompressible Navier-Stokes equations based on Temam's device. J. Comput. Phys., 376:786-816, 2019.

[7] M. Botti, D. A. Di Pietro, and A. Guglielmana. A low-order nonconforming method for linear elasticity on general meshes. Comput. Methods Appl. Mech. Engrg., 354:96-118, 2019.

[8] E. Burman. Ghost penalty. C. R. Math. Acad. Sci. Paris, 348(21-22):1217-1220, 2010.

[9] E. Burman, M. Cicuttin, G. Delay, and A. Ern. An unfitted hybrid high-order method with cell agglomeration for elliptic interface problems. 2019.

[10] E. Burman, S. Claus, P. Hansbo, M. G. Larson, and A. Massing. CutFEM: discretizing geometry and partial differential equations. Internat. J. Numer. Methods Engrg., 104(7):472-501, 2015.

[11] E. Burman and A. Ern. An unfitted hybrid high-order method for elliptic interface problems. SIAM J. Numer. Anal., 56(3):1525-1546, 2018.

[12] E. Cáceres, J. Guzmán, and M. Olshanskii. New stability estimates for an unfitted finite element method for two-phase Stokes problem. ArXiv e-prints, June 2019.

[13] A. P. Calderón. Lebesgue spaces of differentiable functions and distributions. Proc. Sympos. Pure Math., 4:33-49, 1961.

[14] L. Cattaneo, L. Formaggia, G. F. Iori, A. Scotti, and P. Zunino. Stabilized extended finite elements for the approximation of saddle point problems with unfitted interfaces. Calcolo, 52(2):123-152, 2015.

[15] M. Cicuttin, D. A. Di Pietro, and A. Ern. Implementation of discontinuous skeletal methods on arbitrarydimensional, polytopal meshes using generic programming. J. Comput. Appl. Math., 344:852-874, 2018. 
[16] B. Cockburn, D. A. Di Pietro, and A. Ern. Bridging the Hybrid High-Order and Hybridizable Discontinuous Galerkin methods. ESAIM: Math. Model Numer. Anal. (M2AN), 50(3):635-650, 2016.

[17] F. de Prenter, C. Lehrenfeld, and A. Massing. A note on the stability parameter in Nitsche's method for unfitted boundary value problems. Comput. Math. Appl., 75(12):4322-4336, 2018.

[18] D. A. Di Pietro and J. Droniou. The Hybrid High-Order Method for Polytopal Meshes. Design, Analysis, and Applications., volume 19 of Modeling, Simulation \& Applications. Springer International Publishing, Berlin, 2020.

[19] D. A. Di Pietro and A. Ern. A Hybrid High-Order locking-free method for linear elasticity on general meshes. Comput. Meth. Appl. Mech. Engrg., 283:1-21, 2015.

[20] D. A. Di Pietro, A. Ern, and S. Lemaire. An arbitrary-order and compact-stencil discretization of diffusion on general meshes based on local reconstruction operators. Comput. Meth. Appl. Math., 14(4):461-472, 2014 .

[21] Daniele A. Di Pietro, Alexandre Ern, Alexander Linke, and Friedhelm Schieweck. A discontinuous skeletal method for the viscosity-dependent Stokes problem. Comput. Methods Appl. Mech. Engrg., 306:175-195, 2016 .

[22] S. Groß and A. Reusken. An extended pressure finite element space for two-phase incompressible flows with surface tension. J. Comput. Phys., 224(1):40-58, 2007.

[23] A. Hansbo and P. Hansbo. An unfitted finite element method, based on Nitsche's method, for elliptic interface problems. Comput. Methods Appl. Mech. Engrg., 191(47-48):5537-5552, 2002.

[24] P. Hansbo, M. G. Larson, and S. Zahedi. A cut finite element method for a Stokes interface problem. Appl. Numer. Math., 85:90-114, 2014.

[25] F. Heimann, C. Engwer, O. Ippisch, and P. Bastian. An unfitted interior penalty discontinuous Galerkin method for incompressible Navier-Stokes two-phase flow. Internat. J. Numer. Methods Fluids, 71(3):269293,2013

[26] C. O. Horgan. Korn's inequalities and their applications in continuum mechanics. SIAM Rev., 37(4):491511,1995 .

[27] H. Ji and Q. Zhang. A simple finite element method for Stokes flows with surface tension using unfitted meshes. Internat. J. Numer. Methods Fluids, 81(2):87-103, 2016.

[28] A. Johansson and M. G. Larson. A high order discontinuous Galerkin Nitsche method for elliptic problems with fictitious boundary. Numer. Math., 123(4):607-628, 2013.

[29] M. Kirchhart, S. Gross, and A. Reusken. Analysis of an XFEM discretization for Stokes interface problems. SIAM J. Sci. Comput., 38(2):A1019-A1043, 2016.

[30] C. Lehrenfeld and A. Reusken. Analysis of a high-order unfitted finite element method for elliptic interface problems. IMA J. Numer. Anal., 38(3):1351-1387, 2018.

[31] J. Nitsche. Über ein Variationsprinzip zur Lösung von Dirichlet-Problemen bei Verwendung von Teilräumen, die keinen Randbedingungen unterworfen sind. Abh. Math. Sem. Univ. Hamburg, 36:9-15, 1971. Collection of articles dedicated to Lothar Collatz on his sixtieth birthday.

[32] N. Pignet. Hybrid High-Order methods for nonlinear solid mechanics. PhD thesis, ENPC - École des Ponts ParisTech, 2019.

[33] A. Reusken. Analysis of an extended pressure finite element space for two-phase incompressible flows. Comput. Vis. Sci., 11(4-6):293-305, 2008.

[34] W. E. H. Sollie, O. Bokhove, and J. J. W. van der Vegt. Space-time discontinuous Galerkin finite element method for two-fluid flows. J. Comput. Phys., 230(3):789-817, 2011.

[35] E. M. Stein. Singular Integrals and Differentiability Properties of Functions. Princeton, NJ, Princeton University Press, 1970.

[36] Q. Wang and J. Chen. A new unfitted stabilized Nitsche's finite element method for Stokes interface problems. Comput. Math. Appl., 70(5):820-834, 2015. 